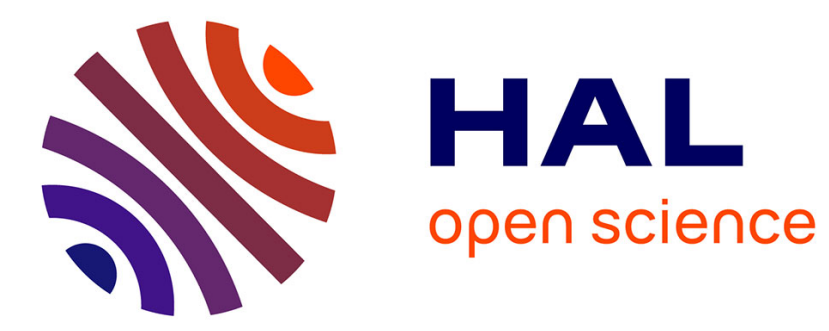

\title{
Multiscale cohesive zone model for propagation of segmented crack fronts in mode I+III fracture
}

\author{
Jean-Baptiste Leblond, Veronique Lazarus, Alain Karma
}

\section{To cite this version:}

Jean-Baptiste Leblond, Veronique Lazarus, Alain Karma. Multiscale cohesive zone model for propagation of segmented crack fronts in mode I+III fracture. International Journal of Fracture, 2015, pp.1-23. 10.1007/s10704-015-0001-x . hal-01130209

\section{HAL Id: hal-01130209 \\ https://hal.sorbonne-universite.fr/hal-01130209}

Submitted on 11 Mar 2015

HAL is a multi-disciplinary open access archive for the deposit and dissemination of scientific research documents, whether they are published or not. The documents may come from teaching and research institutions in France or abroad, or from public or private research centers.
L'archive ouverte pluridisciplinaire HAL, est destinée au dépôt et à la diffusion de documents scientifiques de niveau recherche, publiés ou non, émanant des établissements d'enseignement et de recherche français ou étrangers, des laboratoires publics ou privés. 


\title{
Multiscale cohesive zone model for propagation of segmented crack fronts in mode I+III fracture
}

\author{
Jean-Baptiste Leblond ${ }^{1 *}$, Véronique Lazarus ${ }^{2}$, Alain Karma ${ }^{3}$ \\ ${ }^{1}$ Sorbonne Universités, UPMC Univ Paris 06, CNRS, UMR 7190, Institut Jean Le Rond \\ d'Alembert, F-75005, Paris, France \\ ${ }^{2}$ Univ Paris-Sud, CNRS, UMR 7608, Lab FAST, F-91405, Orsay, France \\ ${ }^{3}$ Physics Department and Center for Interdisciplinary Research on Complex Systems, \\ Northeastern University, Boston, MA 02115, USA
}

\begin{abstract}
Quasistatic crack propagation in mixed-mode I+III fracture is widely observed to be unstable, the instability being characterized by the segmentation of the parent crack into a periodic array of daughter cracks shaped as flat facets rotated towards the principal stress axis. While there has been recent progress to characterize this instability, no global theory is presently available to describe all aspects of the propagation of the segmented front, including both "local" features like the angle of rotation of the facets and the ratio of their width to their spacing, and "global" ones like the effective energy-release-rate of the segmented crack front and the tendency of the facets to coarsen. This paper embarks on the development of such a theory, based on the assumption that the spacing of the facets is much smaller than their length, and asymptotic matching of outer and inner solutions for the mechanical fields on scales comparable to the facet length and spacing, respectively. The inner problem is shown to reduce to a $2 \mathrm{D}$ linear elastic fracture mechanics problem in the plane perpendicular to the crack propagation axis. The solution of this problem is used to develop an effective cohesive zone description of the crack front on a scale much larger than the facet spacing. Such a description leads to a system of 1D integral equations for the outer mechanical fields on the cohesive zone, which may be solved numerically. Numerical examples are given that notably illustrate the prediction of the effective energy-release-rate of the segmented crack front in terms of the various geometrical parameters; this energy-release-rate is predicted to be smaller for a segmented front than for the parent planar front, with the conclusion that segmentation acts as a toughening mechanism. Implications upon the phenomenon of facet coarsening are also briefly discussed.
\end{abstract}

Keywords : crack propagation, mode I+III, tilted facets, cohesive zone model, matched asymptotic expansions, integral equations

$\bar{*}$ Corresponding author. 


\section{Introduction}

The instability of coplanar crack propagation in mixed-mode I+III is well documented experimentally: see for instance the works of Sommer (1969); Knauss (1970); Palaniswamy and Knauss (1975); Hourlier and Pineau (1979); Pollard et al. (1982); Suresh and Tschegg (1987); Pollard and Aydin (1988); Yates and Miller (1989); Cooke and Pollard (1996); Lazarus (1997); Lazarus et al. (2001b, 2008); Lin et al. (2010); Goldstein and Osipenko (2012); Pham and Ravi-Chandar (2014); Ronsin et al. (2014), to name just a few. A systematic observation is that the crack propagates through formation of small fracture facets abruptly or gradually rotating about the direction of propagation. There are two types of facets (Hourlier and Pineau, 1979): "type A" ones rotating in such a way that the local stress intensity factor (SIF) of mode I increases with the distance of propagation while that of mode III decreases, and "type B" ones, rotating oppositely; type A facets are formed preferentially and propagate ahead of type B ones, which are even frequently totally absent. It is also commonly observed that facets tend to "coarsen" in time through a process of coalescence of neighboring facets, each "winning" one tending to absorb its "losing" neighbors.

A few years ago, Pons and Karma (2010) performed numerical simulations of crack propagation in mode I+III, based on a "phase field" model developed by Karma et al. (2001) including a phenomenological description of failure mechanisms in the process zone around the crack front. These simulations reproduced many aspects of non-coplanar crack propagation in mode I+III in a convincing way. Furthermore, by an asymptotic analysis of Karma et al. (2001)'s phase field model in the limit where the process zone size is much smaller than all other dimensions, Hakim and Karma (2009) showed that quasistatic crack propagation in isotropic media is governed, within this model, by a combination of a Griffith (1921) - like condition of uniform energy-release-rate and a Goldstein and Salganik (1974) - like condition of zero mode II SIF. This analysis, combined with the results of Pons and Karma (2010)'s numerical simulations, suggested that crack propagation in mixed-mode I+III fracture should be tractable within the classical framework of linear elastic fracture mechanics (LEFM), by enforcing these conditions along the crack front.

This was the motivation for Leblond et al. (2011)'s recent linear stability analysis of crack propagation in mode I+III. Using first-order analyses of Gao and Rice (1986) and Movchan et al. (1998) of in-plane and out-of-plane perturbations of a semi-infinite crack, Leblond et al. (2011) computed analytically the exponential amplification rate of both types of perturbations, which are inherently coupled when the conditions of constant energy-release-rate and zero mode II SIF are simultaneously satisfied along the crack front. This analysis predicted that mixed-mode I+III crack propagation is linearly unstable for values of the ratio $K_{I I I}^{(0)} / K_{I}^{(0)}$ of the mode III to mode I unperturbed SIF larger than some threshold $\left(K_{I I I}^{(0)} / K_{I}^{(0)}\right)_{\text {cr }}$ depending on Poisson's ratio $\nu$, and that the unstable mode corresponds to a helical deformation of the crack front, consistent with Pons and Karma (2010)'s phase-field simulations.

One limitation of this analysis, however, is that mode I+III fracture is observed experimentally to be generically unstable for values of $K_{I I I}^{(0)} / K_{I}^{(0)}$ substantially smaller than 
the threshold $\left(K_{I I I}^{(0)} / K_{I}^{(0)}\right)_{\mathrm{cr}}$. For example, for a typical value of Poisson's ratio for glass $\nu \approx 0.3$, Leblond et al. (2011)'s analysis predicts $\left(K_{I I I}^{(0)} / K_{I}^{(0)}\right)_{\mathrm{cr}} \approx 0.5$ while Sommer (1969)'s experimental studies of mixed-mode I+III fracture of this material showed that stepped fracture surfaces exist for values of $K_{I I I}^{(0)} / K_{I}^{(0)}$ as small as $\sim 0.1$. Furthermore, Pham and Ravi-Chandar (2014)'s more recent experimental studies have led to the conclusion that no threshold exists at all. From a fundamental viewpoint, the results of Leblond et al. (2011)'s linear stability analysis do not contradict the observation of segmented crack fronts for values of $K_{I I I}^{(0)} / K_{I}^{(0)}$ smaller than $\left(K_{I I I}^{(0)} / K_{I}^{(0)}\right)_{\mathrm{cr}}$, insofar as this analysis does not make any prediction about the nonlinear character of the bifurcation from coplanar to non-coplanar propagation. In particular, if the bifurcation is subcritical, propagating crack front solutions in the form of segmented crack fronts could exist in principle for arbitrarily small $K_{I I I}^{(0)} / K_{I}^{(0)}$, including "discontinuous" propagating solutions with type A facets not connected by type B ones. Such configurations have indeed been observed in both phase field simulations (Pons and Karma, 2010) and experiments (Pham and Ravi-Chandar, 2014) and appear to be a generic nonlinear feature of mixed-mode I+III fracture beyond the scope of linear stability analysis.

The possibility of crack front segmentation for values of $K_{I I I}^{(0)} / K_{I}^{(0)}$ below the linear instability threshold is also suggested (albeit not proven) by considering the role of imperfections upon the propagation path, which are present in any realistic setting. Leblond and Lazarus (2015) for instance very recently examined the effect of fracture toughness fluctuations within the initial crack plane. It had already been anticipated by Gao and Rice (1986) that such fluctuations must generate small in-plane undulations of the crack front and therefore, via the coupling of modes II and III for planar crack problems, a small mode II component along this front bound to make it deviate out of the original crack plane. Leblond and Lazarus (2015) completed their analysis by showing that the out-of-plane deviations thus generated can, even for values of $K_{I I I}^{(0)} / K_{I}^{(0)}$ smaller than $\left(K_{I I I}^{(0)} / K_{I}^{(0)}\right)_{\mathrm{cr}}$, be "unstable" in the sense of Cotterell and Rice (1980). Their findings seem to be qualitatively supported by recent experiments of Ronsin et al. (2014) (although the material used in these experiments was hyperelastic rather than linearly elastic).

In view of the above, the present paper makes the reasonable assumption that, over some range of values of $K_{I I I}^{(0)} / K_{I}^{(0)}$ that can be smaller or larger than $\left(K_{I I I}^{(0)} / K_{I}^{(0)}\right)_{\mathrm{cr}}$, mixed-mode I+III fracture fronts consist of disconnected, tilted facets. Based on this assumption, it lays the theoretical foundation for describing the propagation of such fronts within the classical framework of LEFM. A main goal is to devise means to evaluate the mechanical quantities of interest implied; these quantities include both local features (the SIF on the lateral sides of the facets), and global ones (the overall energy-release-rate due to their propagation). Combined with adequate propagation criteria, the theory will permit, in future studies, to predict various aspects of crack propagation in mode I+III in the form of disconnected, tilted facets; again, these aspects will include both local ones like the tilt angle of the facets and the ratio of their width to their spacing, and global ones like the "critical intensity" of the loading promoting propagation and the tendency of the facets to coarsen.

The basis of the theory will consist of assuming the spacing of the facets to be much smaller than their propagation length, and schematizing the region of these facets as 
an effective cohesive zone (CZ) over which the displacement discontinuities are linearly related to the local stresses. Matched asymptotic expansions, using the ratio $d / a$ of the half-spacing of the facets to their length as a small parameter, will be employed to define the linear relations in question, together defining the "constitutive law" of the CZ. Both the "outer" and "inner" views of the problem will be 2D in essence, but in different planes: the outer, unmagnified view will be, in a plane perpendicular to the original front, that of a $2 \mathrm{D}$ crack extended by a CZ beyond its initial tip, whereas the inner, magnified view will be, in a plane perpendicular to the propagation direction, that of a periodic array of inclined cracks.

Use will be made, for this inner problem, of a very recent solution of Leblond and Frelat (2014); this solution, obtained by combining an analytical approach based on Muskhelishvili (1953)'s method and a numerical, finite-element-based one, applies only to values of the ratio $c / d$ of the facet width to the facet spacing smaller than unity and is approximate (although generally accurate). It must be stressed, however, that the model developed is not, in principle, constrained to refer to this specific solution; other, analytical or even purely numerical solutions could be used, if available. ${ }^{1}$ Care will be taken to provide results in a general format permitting to use any solution, even allowing for values of $c / d$ larger than unity.

The paper is organized as follows:

- As a prerequisite, Section 2 complements the solution for a periodic array of inclined cracks by calculating the variations of remote displacements due to presence of the cracks, since this configuration corresponds to the inner view of the problem and these variations are needed to define the constitutive law of the $\mathrm{CZ}$.

- Section 3 then derives the constitutive law of the $\mathrm{CZ}$ through a technique of matched asymptotic expansions, using the ratio $d / a$ as a small parameter. The main output of the procedure is that the relations connecting the displacement discontinuities across the $\mathrm{CZ}$ to the local stresses, in the outer problem, are identical to those connecting the variations of remote displacements due to presence of the cracks to the remote stresses, in the inner problem. They are thus provided by the results of Section 2 .

- Section 4 then derives the CZ model itself. This is done in several steps, considering now the sole outer problem, the presence of the smaller scale geometric structure being henceforward accounted for through the constitutive law of the CZ:

- Subsection 4.1 first proposes an approximate, "self-consistent" modification of the constitutive law established in Section 3, aimed at correcting some shortcomings of the matched asymptotic expansion procedure near the endpoints of the CZ.

- Subsection 4.2 then establishes 1D integral equations satisfied by the unknown tractions on the $\mathrm{CZ}$, by writing the classical integral expressions of the displacement discontinuities across the CZ in terms of these tractions, and eliminating the discontinuities between these expressions and the constitutive law of the CZ.

- Subsection 4.3 expounds, for future reference but without further insistence on the topic, a heuristic extension of the integral equations accounting for the phenomenon of facet coarsening.

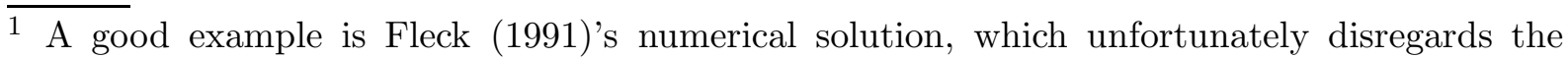
influence of the stress parallel to the crack front. 
- Subsection 4.4 presents a numerical method of solution of the integral equations (in their simpler version).

- Subsection 4.5 finally presents a few numerical illustrations.

- Section 5 briefly discusses how these results may be applied, in future studies, to the theoretical prediction of various aspects of crack propagation in mode I+III in the form of disconnected, tilted facets, including both local features (tilt angle of the facets, ratio of their width to their spacing) and global ones (critical intensity of the loading promoting propagation, tendency of the facets to coarsen).

\section{Variations of remote displacements due to an array of tilted facets}

The first task is to complete the solution for an infinite elastic $2 \mathrm{D}$ body containing a periodic array of inclined cracks, by calculating the variations of remote displacements due to presence of the cracks.

\subsection{Problem considered and outline of the method of solution}

The problem considered (Figure 1) is that of an infinite plate made of some homogeneous and isotropic elastic material and containing a periodic array of identical cracks. The centers of these cracks are aligned on the horizontal axis $O x_{1}$ and the cracks are inclined at an angle $\alpha$ over it. The projected length of the cracks onto the axis $O x_{1}$ is denoted $2 c$, and the distance between their successive centers (the geometric period) $2 d$. The plate is loaded in conditions of plane strain or generalized plane strain ${ }^{2}$ through uniform remote stresses $\sigma_{11}^{\infty}, \sigma_{22}^{\infty}, \sigma_{12}^{\infty}$. The cracks are traction-free and there are no body forces.

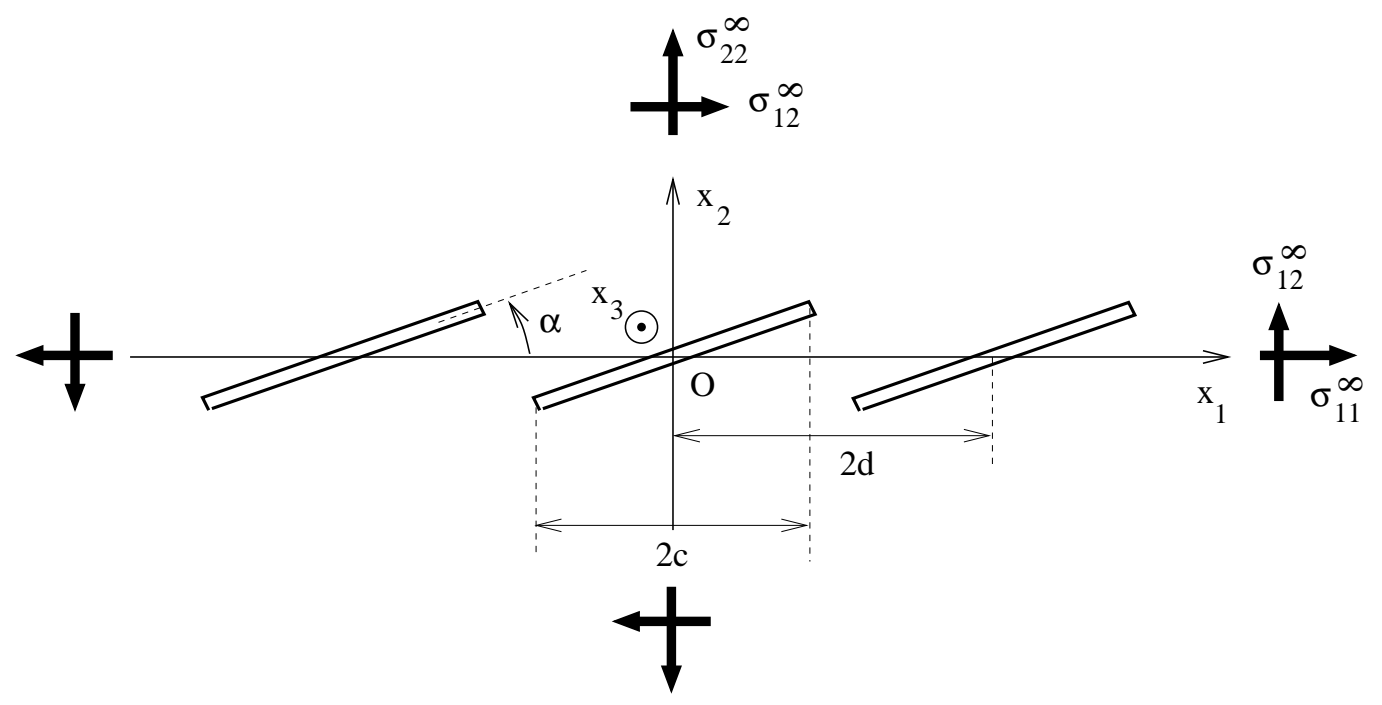

Fig. 1. A periodic array of inclined cracks in an infinite 2D body

$\overline{2}$ This makes no difference on the results, because the difference between the two situations corresponds to a mere uniform stress field $\sigma_{33}$, which has no influence whatsoever on the SIF. 
Our goal here is to obtain the relations connecting the variations of the remote displacements due to presence of the cracks to the remote stresses. To achieve it, we shall introduce, adopting "Voigt-like" notations, the set of load parameters

$$
\left\{\begin{array}{l}
Q_{1} \equiv \sigma_{11}^{\infty} \\
Q_{2} \equiv \sigma_{22}^{\infty} \\
Q_{3} \equiv \sigma_{12}^{\infty}
\end{array}\right.
$$

The associated conjugate parameters $q_{\lambda}, \lambda=1,2,3$ are defined by the formula

$$
\mathcal{P}^{e}=Q_{\lambda} \dot{q}_{\lambda}
$$

where $\mathcal{P}^{e}$ denotes the virtual power of external forces arising from arbitrary virtual velocities $\dot{q}_{\lambda}$, and Einstein's implicit summation convention is used for the index $\lambda$. The parameters $q_{\lambda}$ are obviously linked to the remote displacements; the relations implied will be derived in detail in Subsection 2.2.

The (symmetric) compliance coefficients $\mathcal{C}_{\lambda \mu}, \lambda, \mu=1,2,3$ are defined by the formula

$$
q_{\lambda}=\mathcal{C}_{\lambda \mu} Q_{\mu}
$$

It immediately follows from there, $\Delta f$ denoting the variation of any quantity $f$ due to presence of the cracks, that

$$
\Delta q_{\lambda}=\Delta \mathcal{C}_{\lambda \mu} Q_{\mu}
$$

Formula (4) provides the desired variations of the remote displacements, provided that the variations of the compliance coefficients are themselves known.

The expressions of the latter variations will be derived in Subsection 2.3 by using the well-known compliance formula:

$$
g=\frac{1}{2} \frac{\mathrm{d} \mathcal{C}_{\lambda \mu}}{\mathrm{d} L} Q_{\lambda} Q_{\mu}
$$

where $g$ denotes the energy-release-rate and $L$ the length of the crack. The expression of $g$ here will itself be deduced, via Irwin's classical formula, from the expressions of the 2D stress SIF $k_{I}, k_{I I}$. In Leblond and Frelat (2014)'s work, these SIF were written in the form

$$
\left\{\begin{array}{l}
k_{I} \equiv \sqrt{2 d \tan \left(\frac{\pi c}{2 d}\right)}\left[F_{11}^{I}\left(\frac{c}{d}, \alpha\right) \sigma_{11}^{\infty}+F_{22}^{I}\left(\frac{c}{d}, \alpha\right) \sigma_{22}^{\infty}+F_{12}^{I}\left(\frac{c}{d}, \alpha\right) \sigma_{12}^{\infty}\right] \\
k_{I I} \equiv \sqrt{2 d \tan \left(\frac{\pi c}{2 d}\right)}\left[F_{11}^{I I}\left(\frac{c}{d}, \alpha\right) \sigma_{11}^{\infty}+F_{22}^{I I}\left(\frac{c}{d}, \alpha\right) \sigma_{22}^{\infty}+F_{12}^{I I}\left(\frac{c}{d}, \alpha\right) \sigma_{12}^{\infty}\right]
\end{array}\right.
$$

where the factor $\sqrt{2 d \tan \left(\frac{\pi c}{2 d}\right)}$ was introduced conventionally in reference to the classical solution for a zero $\alpha$ (collinear cracks), see Koiter (1959); and approximate expressions, recalled in Appendix $\mathrm{A}$, of the functions $F_{i j}^{p}$ applicable to values of $c / d$ smaller than unity were proposed. 
A final remark is that Fleck (1991) derived the variations of the remote displacements due to presence of the cracks in a similar problem; however the method of solution proposed here is somewhat more direct, and in addition incorporates the influence of the stress $\sigma_{11}^{\infty}$ which the very method employed by this author prohibited to consider.

\subsection{Domain analyzed, boundary conditions and expressions of conjugate parameters}

The load parameters $Q_{\lambda}$ considered and the associated conjugate parameters $q_{\lambda}$ pertain to a rectangular domain $-d \leq x_{1} \leq d,-H \leq x_{2} \leq H$ where $H$ is a very large real number (Figure 2). The right, left, top and bottom boundaries of this domain will be denoted $\Gamma^{R}$, $\Gamma^{L}, \Gamma^{T}$ and $\Gamma^{B}$, respectively.

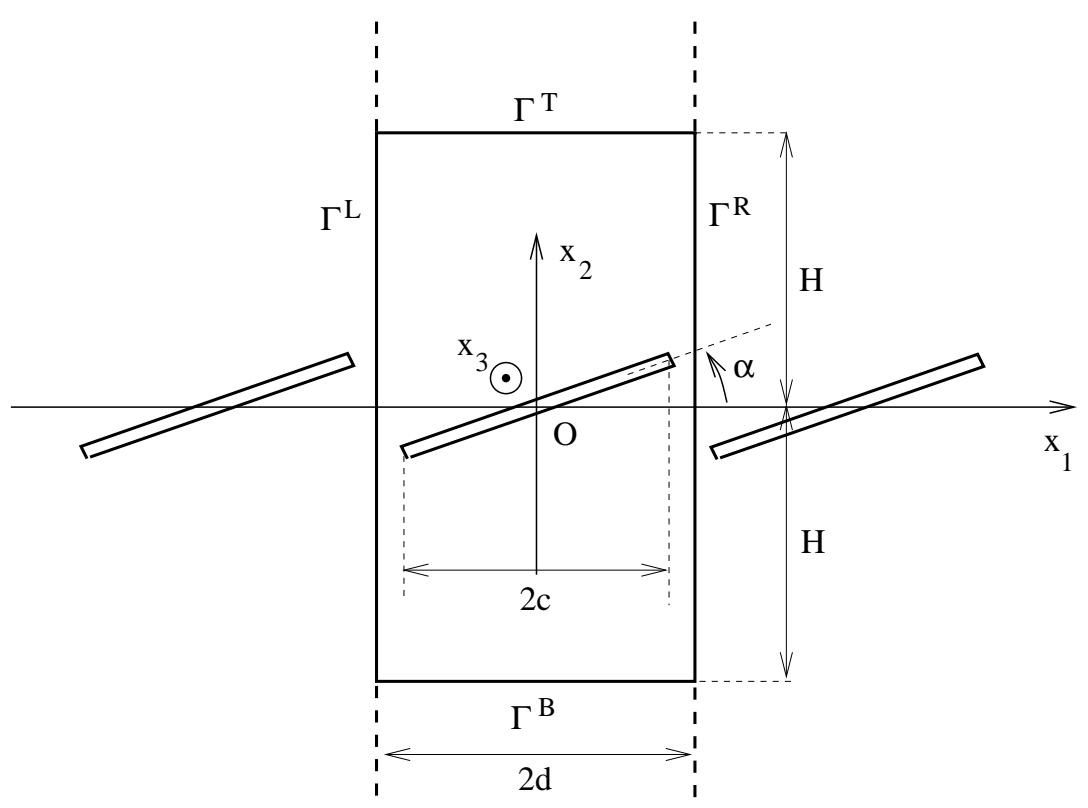

Fig. 2. The domain analyzed

The kinematic conditions prevailing on the right and left boundaries are of periodic type:

$$
\mathbf{u}=\left\{\begin{array}{l}
d\left(\boldsymbol{\epsilon}^{\infty}+\boldsymbol{\omega}^{\infty}\right) \cdot \mathbf{e}_{1}+\widetilde{\mathbf{u}} \text { on } \Gamma^{R} \\
-d\left(\boldsymbol{\epsilon}^{\infty}+\boldsymbol{\omega}^{\infty}\right) \cdot \mathbf{e}_{1}+\widetilde{\mathbf{u}} \text { on } \Gamma^{L}
\end{array}\right.
$$

where $\mathbf{u}$ denotes the local displacement-vector, $\boldsymbol{\epsilon}^{\infty}$ and $\boldsymbol{\omega}^{\infty}$ the remote strain and rotation tensors (symmetric and antisymmetric parts of the gradient of displacement), $\mathbf{e}_{1}$ the unit vector parallel to the direction $x_{1}$, and $\widetilde{\mathbf{u}}$ a function of $x_{1}$ and $x_{2}$ periodic in $x_{1}$, of period $2 d$. On the other hand the kinematic conditions on the top and bottom boundaries are of homogeneous strain type:

$$
\mathbf{u}= \begin{cases}H\left(\boldsymbol{\epsilon}^{\infty}+\boldsymbol{\omega}^{\infty}\right) \cdot \mathbf{e}_{2} & \text { on } \Gamma^{T} \\ -H\left(\boldsymbol{\epsilon}^{\infty}+\boldsymbol{\omega}^{\infty}\right) \cdot \mathbf{e}_{2} & \text { on } \Gamma^{B}\end{cases}
$$

where $\mathbf{e}_{2}$ denotes the unit vector parallel to the direction $x_{2}$. 
The choice of the remote rotation tensor $\boldsymbol{\omega}^{\infty}$ is of course arbitrary, but must be coherent in the region of the main, original crack and in that of the facets represented by the geometry considered; in other words, the average rotation of the original crack plane $O x_{1} x_{3}$ about the axis $O x_{3}$ must be identical in these two regions. Thus if this rotation is conventionally set to zero in the region of the original crack, it must also be zero in the region of the facets, which leads to the choice

$$
\omega_{12}^{\infty}=-\omega_{21}^{\infty}=\epsilon_{12}^{\infty}
$$

The expression of $\mathcal{P}^{e}$ is obviously, $\boldsymbol{\sigma}$ denoting the local stress tensor,

$$
\begin{aligned}
\mathcal{P}^{e} & =\int_{\Gamma^{R}}\left(\boldsymbol{\sigma} \cdot \mathbf{e}_{1}\right) \cdot \dot{\mathbf{u}} \mathrm{d} x_{2}-\int_{\Gamma^{L}}\left(\boldsymbol{\sigma} \cdot \mathbf{e}_{1}\right) \cdot \dot{\mathbf{u}} \mathrm{d} x_{2}+\int_{\Gamma^{T}}\left(\boldsymbol{\sigma} \cdot \mathbf{e}_{2}\right) \cdot \dot{\mathbf{u}} \mathrm{d} x_{1}-\int_{\Gamma^{B}}\left(\boldsymbol{\sigma} \cdot \mathbf{e}_{2}\right) \cdot \dot{\mathbf{u}} \mathrm{d} x_{1} \\
& =\int_{\Gamma^{R}}\left(\boldsymbol{\sigma} \cdot \mathbf{e}_{1}\right) \cdot[\dot{\mathbf{u}}]_{L}^{R} \mathrm{~d} x_{2}+\int_{\Gamma^{T}}\left(\boldsymbol{\sigma} \cdot \mathbf{e}_{2}\right) \cdot[\dot{\mathbf{u}}]_{B}^{T} \mathrm{~d} x_{1}
\end{aligned}
$$

in this equation account has been taken of the fact that $\boldsymbol{\sigma}$ takes identical values on $\Gamma^{R}$ and $\Gamma^{L}$ because of periodicity, and on $\Gamma^{T}$ and $\Gamma^{B}$ because $H$ is very large; and the symbols

$$
\left\{\begin{array}{l}
{[\mathbf{u}]_{L}^{R} \equiv[\mathbf{u}]_{L}^{R}\left(x_{2}\right) \equiv \mathbf{u}\left(d, x_{2}\right)-\mathbf{u}\left(-d, x_{2}\right)} \\
{[\mathbf{u}]_{B}^{T} \equiv[\mathbf{u}]_{B}^{T}\left(x_{1}\right) \equiv \mathbf{u}\left(x_{1}, H\right)-\mathbf{u}\left(x_{1},-H\right)}
\end{array}\right.
$$

denote the differences of displacements between the right and left boundaries and the top and bottom ones, respectively. Now by equations $(7),(8)$ and (9), the components of these differences of displacements are given by

$$
\left\{\begin{array}{l}
{\left[u_{1}\right]_{L}^{R}=2 d \epsilon_{11}^{\infty}} \\
{\left[u_{2}\right]_{L}^{R}=2 d\left(\epsilon_{21}^{\infty}+\omega_{21}^{\infty}\right)=0}
\end{array} ;\left\{\begin{array}{l}
{\left[u_{1}\right]_{B}^{T}=2 H\left(\epsilon_{12}^{\infty}+\omega_{12}^{\infty}\right)=4 H \epsilon_{12}^{\infty}} \\
{\left[u_{2}\right]_{B}^{T}=2 H \epsilon_{22}^{\infty},}
\end{array}\right.\right.
$$

and it follows that

$$
\mathcal{P}^{e}=2 d \dot{\epsilon}_{11}^{\infty} \int_{\Gamma^{R}} \sigma_{11} \mathrm{~d} x_{2}+4 H \dot{\epsilon}_{12}^{\infty} \int_{\Gamma^{T}} \sigma_{12} \mathrm{~d} x_{1}+2 H \dot{\epsilon}_{22}^{\infty} \int_{\Gamma^{T}} \sigma_{22} \mathrm{~d} x_{1} .
$$

But in the limit $H \rightarrow+\infty, \boldsymbol{\sigma}$ becomes identical to $\boldsymbol{\sigma}^{\infty}$ on $\Gamma^{T}$, and on an ever increasing part of $\Gamma^{R}$. Therefore in this limit

$$
\mathcal{P}^{e}=4 d H \sigma_{11}^{\infty} \dot{\epsilon}_{11}^{\infty}+4 d H \sigma_{22}^{\infty} \dot{\epsilon}_{22}^{\infty}+8 d H \sigma_{12}^{\infty} \dot{\epsilon}_{12}^{\infty}=4 d H Q_{1} \dot{\epsilon}_{11}^{\infty}+4 d H Q_{2} \dot{\epsilon}_{22}^{\infty}+8 d H Q_{3} \dot{\epsilon}_{12}^{\infty}
$$

where the definition (1) of the load parameters $Q_{\lambda}$ has been used. Comparison with the definition (2) of the conjugate parameters $q_{\lambda}$ then reveals that

$$
\left\{\begin{array}{l}
q_{1} \equiv 4 d H \epsilon_{11}^{\infty}=2 H\left[u_{1}\right]_{L}^{R} \\
q_{2} \equiv 4 d H \epsilon_{22}^{\infty}=2 d\left[u_{2}\right]_{B}^{T} \\
q_{3} \equiv 8 d H \epsilon_{12}^{\infty}=2 d\left[u_{1}\right]_{B}^{T}
\end{array}\right.
$$


where equations (11) have been used. It then follows from there and equations (4) that

$$
\left\{\begin{array}{l}
\Delta\left[u_{1}\right]_{L}^{R}=\Delta q_{1} /(2 H)=\Delta \mathcal{C}_{1 \mu} Q_{\mu} /(2 H) \\
\Delta\left[u_{1}\right]_{B}^{T}=\Delta q_{3} /(2 d)=\Delta \mathcal{C}_{3 \mu} Q_{\mu} /(2 d) \\
\Delta\left[u_{2}\right]_{B}^{T}=\Delta q_{2} /(2 d)=\Delta \mathcal{C}_{2 \mu} Q_{\mu} /(2 d) .
\end{array}\right.
$$

\subsection{Application of the compliance formula}

To now calculate the variations $\Delta \mathcal{C}_{\lambda \mu}$ of the compliance coefficients, we combine the compliance formula (5) and Irwin's expression of $g$; since $L=2 c / \cos \alpha$ (see Figure 2), we get

$$
\frac{\mathrm{d} \mathcal{C}_{\lambda \mu}}{\mathrm{d} c} Q_{\lambda} Q_{\mu}=\frac{4}{\cos \alpha} g=\frac{4}{\cos \alpha} \frac{1-\nu^{2}}{E}\left(k_{I}^{2}+k_{I I}^{2}\right)
$$

where $E$ and $\nu$ denote Young's modulus and Poisson's ratio, respectively. Now if one adopts, in line with equations (1), the Voigt-like notations

$$
\left\{\begin{array}{l}
F_{1}^{p}(c / d, \alpha) \equiv F_{11}^{p}(c / d, \alpha) \\
F_{2}^{p}(c / d, \alpha) \equiv F_{22}^{p}(c / d, \alpha) \\
F_{3}^{p}(c / d, \alpha) \equiv F_{12}^{p}(c / d, \alpha)
\end{array} \quad(p=I, I I),\right.
$$

the expression (6) of the SIF takes the form

$$
k_{p}=\sqrt{2 d \tan \left(\frac{\pi c}{2 d}\right)} F_{\lambda}^{p}\left(\frac{c}{d}, \alpha\right) Q_{\lambda}
$$

so that the preceding expression of $\frac{\mathrm{d} \mathcal{C}_{\lambda \mu}}{\mathrm{d} c} Q_{\lambda} Q_{\mu}$ may be rewritten as

$$
\frac{\mathrm{d} \mathcal{C}_{\lambda \mu}}{\mathrm{d} c} Q_{\lambda} Q_{\mu}=\frac{8 d}{\cos \alpha} \frac{1-\nu^{2}}{E} \tan \left(\frac{\pi c}{2 d}\right) F_{\lambda}^{p}\left(\frac{c}{d}, \alpha\right) F_{\mu}^{p}\left(\frac{c}{d}, \alpha\right) Q_{\lambda} Q_{\mu}
$$

where Einstein's implicit summation convention is used for the index $p$. It follows, since this equality holds for all possible values of the load parameters, that

$$
\frac{\mathrm{d} \mathcal{C}_{\lambda \mu}}{\mathrm{d} c}=\frac{8 d}{\cos \alpha} \frac{1-\nu^{2}}{E} \tan \left(\frac{\pi c}{2 d}\right) F_{\lambda}^{p}\left(\frac{c}{d}, \alpha\right) F_{\mu}^{p}\left(\frac{c}{d}, \alpha\right)
$$

for every $\lambda$ and $\mu$.

Integrating equation (15) between 0 and $c$, one gets the variations of the compliance coefficients looked for:

$$
\Delta \mathcal{C}_{\lambda \mu}=\frac{8 d}{\cos \alpha} \frac{1-\nu^{2}}{E} \int_{0}^{c} \tan \left(\frac{\pi c^{\prime}}{2 d}\right) F_{\lambda}^{p}\left(\frac{c^{\prime}}{d}, \alpha\right) F_{\mu}^{p}\left(\frac{c^{\prime}}{d}, \alpha\right) \mathrm{d} c^{\prime} .
$$


Upon use of the changes of variables

$$
x \equiv \frac{c}{d} \quad ; \quad x^{\prime} \equiv \frac{c^{\prime}}{d},
$$

this expression becomes

$$
\Delta \mathcal{C}_{\lambda \mu}=\frac{8\left(1-\nu^{2}\right) d^{2}}{E} \mathcal{A}_{\lambda \mu}
$$

where the (symmetric) dimensionless coefficients $\mathcal{A}_{\lambda \mu}$ are defined by

$$
\mathcal{A}_{\lambda \mu} \equiv \mathcal{A}_{\lambda \mu}(x, \alpha) \equiv \frac{1}{\cos \alpha} \int_{0}^{x} \tan \left(\frac{\pi x^{\prime}}{2}\right) F_{\lambda}^{p}\left(x^{\prime}, \alpha\right) F_{\mu}^{p}\left(x^{\prime}, \alpha\right) \mathrm{d} x^{\prime} .
$$

Approximate values of these coefficients, based on Leblond and Frelat (2014)'s approximate expressions of the functions $F_{\lambda}^{p}$ recalled in Appendix A, are provided in Appendix $\mathrm{B}$, and will be used to provide some final numerical illustrations. It must however be stressed that none of the theoretical formulae derived below will make any reference to them, which should make it very easy to replace them with more accurate ones, if and when they become available.

Equations (17) and (18) make it clear that in contrast to the compliance coefficients $\mathcal{C}_{\lambda \mu}$ themselves, their variations $\Delta \mathcal{C}_{\lambda \mu}$ are independent of $H$. Therefore in the limit $H \rightarrow+\infty$, equation $(13)_{1}$ implies that the variation of displacement $\Delta\left[u_{1}\right]_{L}^{R}$ becomes zero: this means that presence of the crack does not modify the horizontal strain of the vertical band containing it, which is a natural result since this band is infinite. This is not true, however, for the variations of displacements $\Delta\left[u_{1}\right]_{B}^{T}$ and $\Delta\left[u_{1}\right]_{B}^{T}$; by equations $(13)_{2,3}$ and (17), these variations are given, henceforth dropping the symbols $T$ and $B$ for simplicity ${ }^{3}$, by

$$
\left\{\begin{array}{l}
\Delta\left[u_{1}\right]=\frac{4\left(1-\nu^{2}\right) d}{E} \mathcal{A}_{3 \mu} Q_{\mu}=\frac{4\left(1-\nu^{2}\right) d}{E}\left(\mathcal{A}_{31} \sigma_{11}^{\infty}+\mathcal{A}_{32} \sigma_{22}^{\infty}+\mathcal{A}_{33} \sigma_{12}^{\infty}\right) \\
\Delta\left[u_{2}\right]=\frac{4\left(1-\nu^{2}\right) d}{E} \mathcal{A}_{2 \mu} Q_{\mu}=\frac{4\left(1-\nu^{2}\right) d}{E}\left(\mathcal{A}_{21} \sigma_{11}^{\infty}+\mathcal{A}_{22} \sigma_{22}^{\infty}+\mathcal{A}_{23} \sigma_{12}^{\infty}\right) .
\end{array}\right.
$$

These are the final expressions looked for. Note that the coefficient $\mathcal{A}_{11}$ does not appear here. (But the influence of the stress $\sigma_{11}^{\infty}$ appears through the coefficients $\mathcal{A}_{21}$ and $\mathcal{A}_{31}$ ).

\section{Matched asymptotic expansions}

We now wish to use the preceding solution to derive an approximate model for crack propagation in mode I+III in the form of disconnected tilted facets. The idea is to schematize the region of these facets, ahead of the original crack front, as a CZ on which the displacement jumps are linearly related to the local stresses through relations analogous to (19). The linear relations in question will be determined by assuming the spacing of these facets to be much smaller than their length and using the method of matched asymptotic

$\overline{3}$ This will not raise any ambiguity since no further reference will be made to the variations $\Delta\left[u_{1}\right]_{L}^{R}$ and $\Delta\left[u_{2}\right]_{L}^{R}$, which are both zero. 
expansions, first introduced in solid mechanics, in particular for fracture problems, by Sanchez-Hubert and Sanchez-Palencia (1992) and Leguillon (1993).

More specifically, Figure 3 illustrates the geometry considered. The initially planar crack is loaded in mode I+III, the remote strain parallel to the direction of the crack front being zero. Cartesian axes $O X, O Y, O Z$ are introduced with, following the usual conventions, $O X$ parallel to the direction of propagation, $O Y$ perpendicular to the initial crack plane, and $O Z$ coinciding with the initial crack front. The facets, of type A only, are disconnected and develop from the original front $O Z$ in the direction $X .{ }^{4}$ Their length is denoted $a$ and their stationary tilt angle $\alpha$. Their width, projected onto the axis $O Z$, and their spacing (the geometric period) are denoted $2 c$ and $2 d$ respectively. The length $a$ is considered as fixed whereas both lengths $c$ and $d$ are considered to go to zero, the ratio $c / d$ remaining fixed. The ratio

$$
\eta \equiv \frac{d}{a}
$$

will serve as a small parameter in the matched asymptotic expansion procedure.

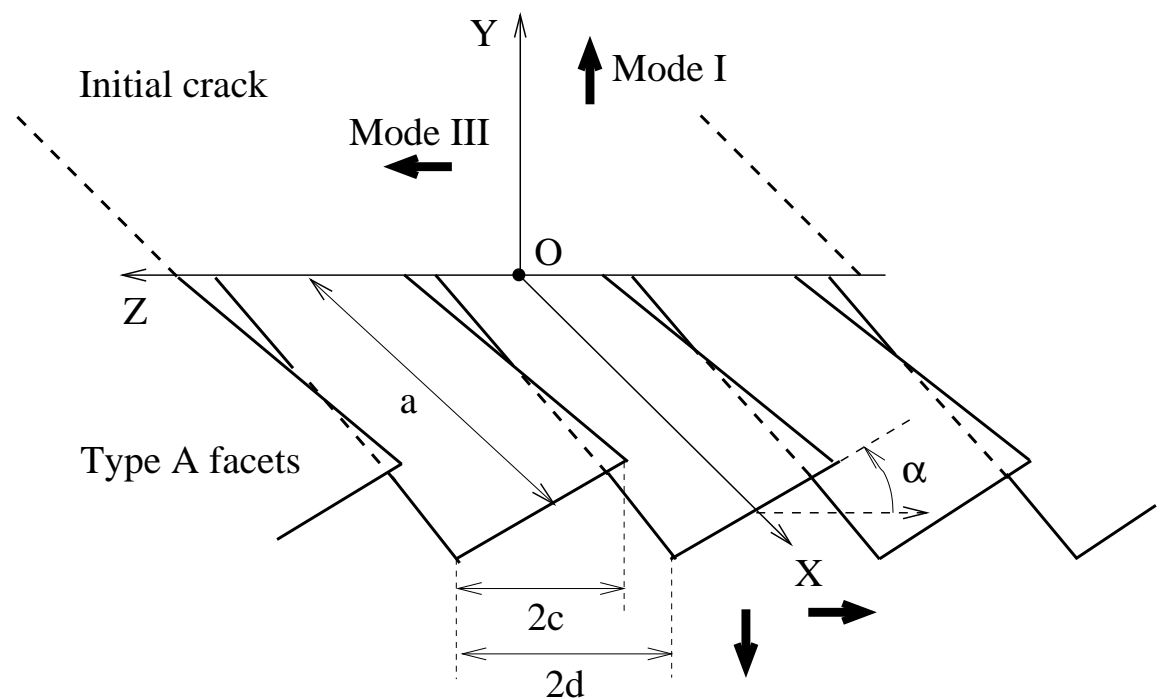

Fig. 3. A mode I+III crack propagating through development of disconnected type A facets

\subsection{Generalities on the outer and inner problems}

Figure 4 provides $2 \mathrm{D}$ views of the geometries of the outer and inner problems.

Figure 4(a) represents the outer problem geometry in the plane $O X Y$. Equations pertaining to this problem will be written, for the sake of coherence with the coordinates of the

$\overline{4}$ Their representation in Figure 3 is only schematic since they should have sharp tips instead of square ones, and reach a stationary orientation after a certain distance of propagation. 


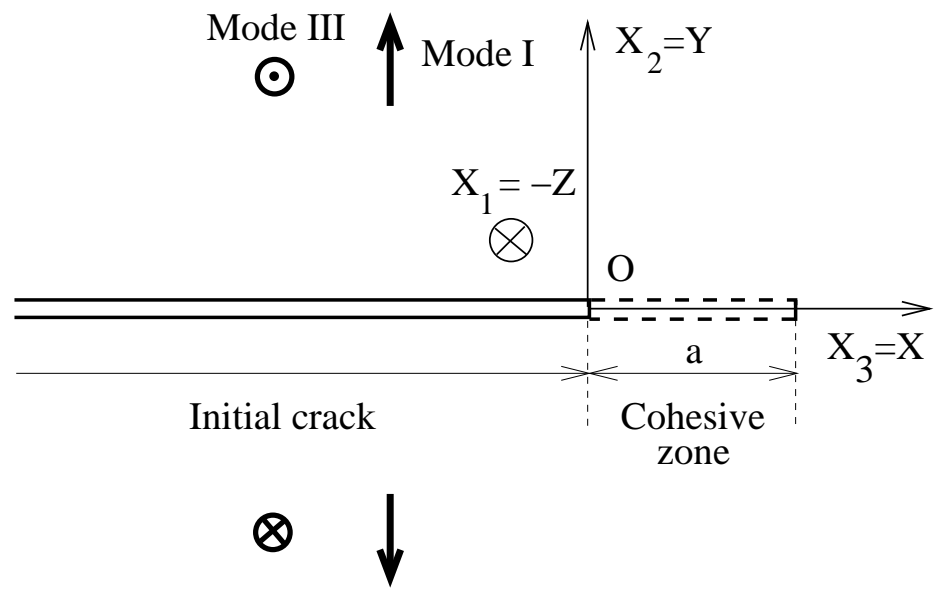

(a) Outer problem

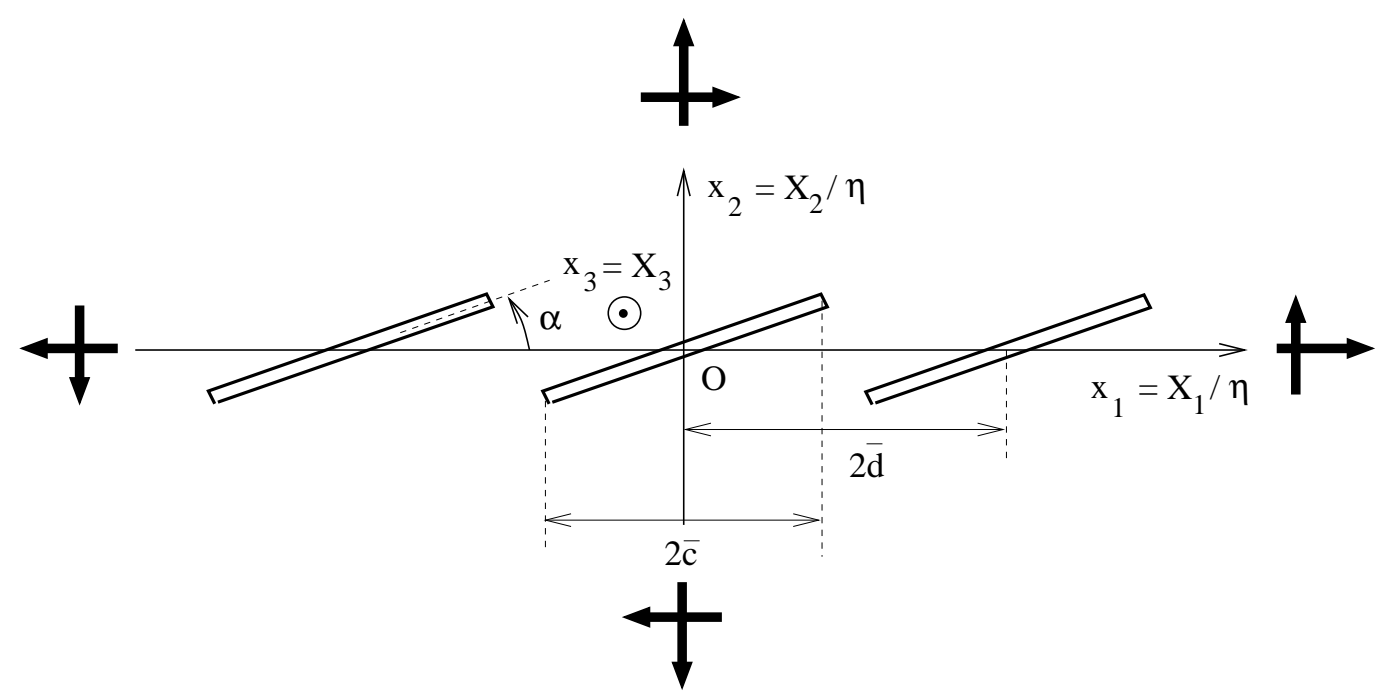

(b) Inner problem

Fig. 4. 2D views of the geometries of the outer and inner problems inner problem, using some coordinates $X_{1}, X_{2}, X_{3}$ defined by

$$
\left\{\begin{array}{l}
X_{1} \equiv-Z \\
X_{2} \equiv Y \\
X_{3} \equiv X
\end{array}\right.
$$

To define the inner problem, an expansion of the sole first two coordinates of the outer problem is performed; that is, one introduces coordinates $x_{1}, x_{2}, x_{3}$ defined by

$$
\left\{\begin{array}{l}
x_{1} \equiv X_{1} / \eta \\
x_{2} \equiv X_{2} / \eta \\
x_{3} \equiv X_{3}
\end{array}\right.
$$

Figure 4(b) represents the inner problem geometry in the plane $O x_{1} x_{2}$. It is identical to 
that of the model problem considered in Section 2 (Figure 1), except for the replacement of the lengths $c$ and $d$ by the new lengths

$$
\bar{c} \equiv \frac{c}{\eta} \quad ; \quad \bar{d} \equiv \frac{d}{\eta}
$$

(which preserves the ratio $c / d$ and thus the coefficients $\mathcal{A}_{\lambda \mu}$, see equations $(16)_{1}$ and (18)).

In the outer problem, the displacement-, strain- and stress fields will be denoted $\mathbf{U}(\mathbf{X})$, $\mathbf{E}(\mathbf{X}), \boldsymbol{\Sigma}(\mathbf{X})$ respectively. In the inner problem, the corresponding fields $\mathbf{u}(\mathbf{x}), \boldsymbol{\epsilon}(\mathbf{x}), \boldsymbol{\sigma}(\mathbf{x})$ will be defined as

$$
\left\{\begin{array}{l}
\mathbf{u}(\mathbf{x}) \equiv \mathbf{U}(\mathbf{X}) \\
\boldsymbol{\epsilon}(\mathbf{x}) \equiv \eta \mathbf{E}(\mathbf{X}) \\
\boldsymbol{\sigma}(\mathbf{x}) \equiv \eta \mathbf{\Sigma}(\mathbf{X})
\end{array}\right.
$$

All fields will be expanded in powers of $\eta$; for instance $\mathbf{U}$ and $\mathbf{u}$ will be written as

$$
\left\{\begin{array}{l}
\mathbf{U}(\mathbf{X})=\sum_{p \geq 0} \eta^{p} \mathbf{U}^{(p)}(\mathbf{X}) \\
\mathbf{u}(\mathbf{x})=\sum_{p \geq 0} \eta^{p} \mathbf{u}^{(p)}(\mathbf{x})
\end{array}\right.
$$

and similarly for $\mathbf{E}$ and $\boldsymbol{\epsilon}, \boldsymbol{\Sigma}$ and $\boldsymbol{\sigma}$.

Introducing Latin indices taking the values 1, 2, 3 and Greek indices taking the sole values 1, 2, and using Einstein's implicit summation convention for all of these indices, the equilibrium equations take the form

$$
\left\{\begin{array}{l}
\frac{\partial \Sigma_{\alpha j}}{\partial X_{j}}=\frac{\partial \Sigma_{\alpha \beta}}{\partial X_{\beta}}+\frac{\partial \Sigma_{\alpha 3}}{\partial X_{3}}=\frac{1}{\eta^{2}} \frac{\partial \sigma_{\alpha \beta}}{\partial x_{\beta}}+\frac{1}{\eta} \frac{\partial \sigma_{\alpha 3}}{\partial x_{3}}=0 \\
\frac{\partial \Sigma_{3 j}}{\partial X_{j}}=\frac{\partial \Sigma_{3 \beta}}{\partial X_{\beta}}+\frac{\partial \Sigma_{33}}{\partial X_{3}}=\frac{1}{\eta^{2}} \frac{\partial \sigma_{3 \beta}}{\partial x_{\beta}}+\frac{1}{\eta} \frac{\partial \sigma_{33}}{\partial x_{3}}=0
\end{array}\right.
$$

from which follows that at every order $p$, these equations read, in the outer and inner problems respectively:

$$
\frac{\partial \Sigma_{i j}^{(p)}}{\partial X_{j}}=0 \quad ; \quad\left\{\begin{array}{l}
\frac{\partial \sigma_{\alpha \beta}^{(p)}}{\partial x_{\beta}}+\frac{\partial \sigma_{\alpha 3}^{(p-1)}}{\partial x_{3}}=0 \\
\frac{\partial \sigma_{3 \beta}^{(p)}}{\partial x_{\beta}}+\frac{\partial \sigma_{33}^{(p-1)}}{\partial x_{3}}=0 .
\end{array}\right.
$$

Note that the equilibrium equations of the inner problem mix stresses of different orders. 
Similarly, the strain components are given by

$$
\left\{\begin{array}{l}
E_{\alpha \beta}=\frac{1}{2}\left(\frac{\partial U_{\alpha}}{\partial X_{\beta}}+\frac{\partial U_{\beta}}{\partial X_{\alpha}}\right)=\frac{1}{2 \eta}\left(\frac{\partial u_{\alpha}}{\partial x_{\beta}}+\frac{\partial u_{\beta}}{\partial x_{\alpha}}\right)=\frac{\epsilon_{\alpha \beta}}{\eta} \\
E_{\alpha 3}=\frac{1}{2}\left(\frac{\partial U_{\alpha}}{\partial X_{3}}+\frac{\partial U_{3}}{\partial X_{\alpha}}\right)=\frac{1}{2}\left(\frac{\partial u_{\alpha}}{\partial x_{3}}+\frac{1}{\eta} \frac{\partial u_{3}}{\partial x_{\alpha}}\right)=\frac{\epsilon_{\alpha 3}}{\eta} \\
E_{33}=\frac{\partial U_{3}}{\partial X_{3}}=\frac{\partial u_{3}}{\partial x_{3}}=\frac{\epsilon_{33}}{\eta}
\end{array}\right.
$$

from which follows that at every order $p$,

$$
E_{i j}^{(p)}=\frac{1}{2}\left(\frac{\partial U_{i}^{(p)}}{\partial X_{j}}+\frac{\partial U_{j}^{(p)}}{\partial X_{i}}\right) ;\left\{\begin{array}{l}
\epsilon_{\alpha \beta}^{(p)}=\frac{1}{2}\left(\frac{\partial u_{\alpha}^{(p)}}{\partial x_{\beta}}+\frac{\partial u_{\beta}^{(p)}}{\partial x_{\alpha}}\right) \\
\epsilon_{\alpha 3}^{(p)}=\frac{1}{2}\left(\frac{\partial u_{\alpha}^{(p-1)}}{\partial x_{3}}+\frac{\partial u_{3}^{(p)}}{\partial x_{\alpha}}\right) \\
\epsilon_{33}^{(p)}=\frac{\partial u_{3}^{(p-1)}}{\partial x_{3}}
\end{array}\right.
$$

where again displacements of different orders are mixed in the inner problem.

In contrast, $\mathbf{C}$ denoting the elastic compliance tensor, the constitutive law

$$
\mathrm{E}=\mathrm{C}: \Sigma \quad \Leftrightarrow \quad \epsilon=\mathrm{C}: \boldsymbol{\sigma}
$$

leads at every order to

$$
\mathbf{E}^{(p)}=\mathbf{C}: \boldsymbol{\Sigma}^{(p)} \quad ; \quad \boldsymbol{\epsilon}^{(p)}=\mathbf{C}: \boldsymbol{\sigma}^{(p)}
$$

where no mixing of quantities of different orders appears.

The displacement fields $\mathbf{U}^{(p)}$ and $\mathbf{u}^{(p)}$ will be expanded in the vicinity of the $\mathrm{CZ}$ and near infinity respectively, in the form

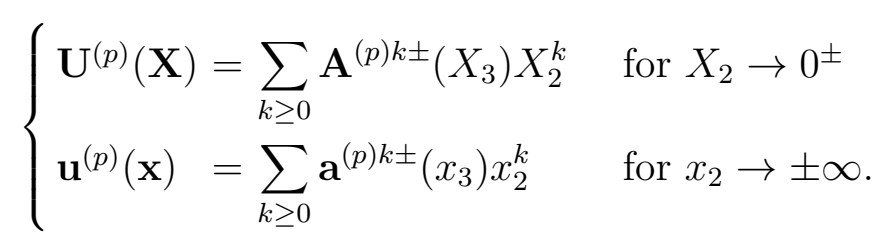

The coefficients $\mathbf{A}^{(p) k \pm}$ here are independent of the coordinate $X_{1}$ because the outer problem has a translatory invariance in the corresponding direction, whereas the coefficients $\mathbf{a}^{(p) k \pm}$ are independent of $x_{1}$ because in the inner problem, the dependence of $\mathbf{u}$ upon this coordinate disappears far from the cracks.

The procedure of matched asymptotic expansions demands that the double expansion of $\mathbf{U}$ in powers of $\eta$ and $X_{2}$ for small values of $X_{2}$,

$$
\mathbf{U}(\mathbf{X})=\sum_{p \geq 0} \sum_{k \geq 0} \eta^{p} \mathbf{A}^{(p) k \pm}\left(X_{3}\right) X_{2}^{k},
$$


be term-to-term identical to the double expansion of $\mathbf{u}$ in powers of $\eta$ and $x_{2}$ for large values of $x_{2}$,

$$
\mathbf{u}(\mathbf{x})=\sum_{p \geq 0} \sum_{k \geq 0} \eta^{p} \mathbf{a}^{(p) k \pm}\left(x_{3}\right) x_{2}^{k}
$$

Since, by equations (22), $\eta^{p} \mathbf{a}^{(p) k \pm}\left(x_{3}\right) x_{2}^{k}=\eta^{p} \mathbf{a}^{(p) k \pm}\left(X_{3}\right)\left(X_{2} / \eta\right)^{k}=\eta^{p-k} \mathbf{a}^{(p) k \pm}\left(X_{3}\right) X_{2}^{k}$, this requires that the matching conditions

$$
\mathbf{A}^{(p-k) k \pm}\left(X_{3}\right)=\mathbf{a}^{(p) k \pm}\left(x_{3}\right) \quad(p \geq 0, k \geq 0)
$$

be satisfied for every $X_{3}=x_{3}$. These conditions imply in particular, since the expansion of $\mathbf{U}$ obviously cannot contain any negative power of $\eta$, that coefficients $\mathbf{a}^{(p) k \pm}\left(x_{3}\right)$ having $k>p$ must necessarily be zero; in other words $\mathbf{u}^{(p)}(\mathbf{x})$ is necessarily $O\left(x_{2}^{p}\right)$ at infinity.

\subsection{Outer problem at order 0}

In the outer problem at order 0 , that is in the limit where the geometrical period $2 d$ goes to zero, the length $a$ of the facets being fixed, equations (19) show that the effect of the facets, proportional to $d$, completely disappears. To take a picturesque image, the situation is analogous to the buttoning of a tight shirt: the larger the number of buttons, the less open the shirt between two consecutive buttons; and in the limit of a continuous zip, the opening becomes nil.

The problem is thus simply that of the initial planar crack without any facets, loaded in mode I+III. The mechanical fields are asymptotically governed near the initial crack front $O X_{1}$ by the SIF $K_{I}^{(0)}, K_{I I I}^{(0)}$. Near the CZ, the displacement field $\mathbf{U}^{(0)}$ is given by equation $(29)_{1}$, where the coefficients $\mathbf{A}^{(0) k \pm}$ are identical in the upper $(+)$ and lower $(-)$ half-spaces because of regularity of the solution across the plane $X_{2}=0: \mathbf{A}^{(0) k+}\left(X_{3}\right)=$ $\mathbf{A}^{(0) k-}\left(X_{3}\right) \equiv \mathbf{A}^{(0) k}\left(X_{3}\right)$. On the CZ itself, the components $U_{1}^{(0)}$ and $U_{2}^{(0)}$ are obviously zero, so that $A_{1}^{(0) 0}\left(X_{3}\right)=A_{2}^{(0) 0}\left(X_{3}\right)=0$; also, $U_{3}^{(0)}$ is an even function of $X_{2}$ so that $A_{3}^{(0) 1}\left(X_{3}\right)=0$. Therefore the expansion $(29)_{1}$ takes the form, retaining the sole first two terms,

$$
\mathbf{U}^{(0)}(\mathbf{X})=A_{3}^{(0) 0}\left(X_{3}\right) \mathbf{e}_{3}+\left[A_{1}^{(0) 1}\left(X_{3}\right) \mathbf{e}_{1}+A_{2}^{(0) 1}\left(X_{3}\right) \mathbf{e}_{2}\right] X_{2}+O\left(X_{2}^{2}\right) \quad \text { for } X_{2} \rightarrow 0^{ \pm}
$$

Also, the nonzero stress components on the $\mathrm{CZ}$ are given by the classical formulae:

$$
\begin{cases}\Sigma_{22}^{(0) \mathrm{CZ}}=\Sigma_{33}^{(0) \mathrm{CZ}} & =\frac{K_{I}^{(0)}}{\sqrt{2 \pi X_{3}}} \\ \Sigma_{11}^{(0) \mathrm{CZ}}=\nu\left(\Sigma_{22}^{(0) \mathrm{CZ}}+\Sigma_{33}^{(0) \mathrm{CZ}}\right) & =\frac{2 \nu K_{I}^{(0)}}{\sqrt{2 \pi X_{3}}} \\ \Sigma_{12}^{(0) \mathrm{CZ}} & =-\frac{K_{I I I}^{(0)}}{\sqrt{2 \pi X_{3}}}\end{cases}
$$

where the - sign in the last expression arises from the relations (21) connecting the coordinates $X_{1}, X_{2}, X_{3}$ to the original ones $X, Y, Z$. These expressions disregard the influence 
of higher-order terms in the Williams expansion of the stresses, the underlying assumption being that all characteristic distances defined by the far geometry and loading are much larger than the length $a$ of the CZ.

\subsection{Inner problem at order 0}

For the inner problem at order 0 , equations $(26)_{2},(27)_{2}$ and $(28)_{2}$ reduce to

$$
\left\{\begin{array}{l}
\frac{\partial \sigma_{\alpha \beta}^{(0)}}{\partial x_{\beta}}=0 \\
\frac{\partial \sigma_{3 \beta}^{(0)}}{\partial x_{\beta}}=0
\end{array} ;\left\{\begin{array}{l}
\epsilon_{\alpha \beta}^{(0)}=\frac{1}{2}\left(\frac{\partial u_{\alpha}^{(0)}}{\partial x_{\beta}}+\frac{\partial u_{\beta}^{(0)}}{\partial x_{\alpha}}\right) \\
\epsilon_{\alpha 3}^{(0)}=\frac{1}{2} \frac{\partial u_{3}^{(0)}}{\partial x_{\alpha}} \\
\epsilon_{33}^{(0)}=0
\end{array} ; \quad \boldsymbol{\epsilon}^{(0)}=\mathbf{C}: \boldsymbol{\sigma}^{(0)}\right.\right.
$$

and the cracks are traction-free. In addition, equation (30) yields for $p=k=0: \mathbf{A}^{(0) 0}\left(X_{3}\right)=$ $A_{3}^{(0) 0}\left(X_{3}\right) \mathbf{e}_{3}=\mathbf{a}^{(0) 0 \pm}\left(x_{3}\right)$, which implies (since the expansion of $\mathbf{u}^{(0)}$ cannot contain any term in $x_{2}^{k}$ with $k \geq 1$, see above) that $\mathbf{u}^{(0)}$ must satisfy the condition

$$
\lim _{x_{2} \rightarrow \pm \infty} \mathbf{u}^{(0)}(\mathbf{x})=A_{3}^{(0) 0}\left(x_{3}\right) \mathbf{e}_{3} .
$$

The equations of the problem show that it splits, for every value of $x_{3}$, into two independent problems, for the displacement components $\left(u_{1}^{(0)}, u_{2}^{(0)}\right)$ and $u_{3}^{(0)}$ respectively. The problem for $\left(u_{1}^{(0)}, u_{2}^{(0)}\right)$ is homogeneous (zero data), see equation (34) where the right-hand side has no components on the basis vectors $\mathbf{e}_{1}$ and $\mathbf{e}_{2}$; hence its solution is zero. The problem for $u_{3}^{(0)}$ is inhomogeneous (nonzero data) but its solution is trivial: $u_{3}^{(0)}=A_{3}^{(0) 0}\left(x_{3}\right)$; the stresses are nil since the sole nonzero displacement derivative, $\partial u_{3}^{(0)} / \partial x_{3}$, does not enter the expressions of the strains, see equations (33). Therefore the solution reads

$$
\mathbf{u}^{(0)}=A_{3}^{(0) 0}\left(x_{3}\right) \mathbf{e}_{3} \quad ; \quad \boldsymbol{\sigma}^{(0)}=\mathbf{0} .
$$

\subsection{Inner problem at order 1}

For the inner problem at order 1 , equations $(26)_{2},(27)_{2}$ and $(28)_{2}$ read

$$
\left\{\begin{array}{l}
\frac{\partial \sigma_{\alpha \beta}^{(1)}}{\partial x_{\beta}}+\frac{\partial \sigma_{\alpha 3}^{(0)}}{\partial x_{3}}=\frac{\partial \sigma_{\alpha \beta}^{(1)}}{\partial x_{\beta}}=0 \\
\frac{\partial \sigma_{3 \beta}^{(1)}}{\partial x_{\beta}}+\frac{\partial \sigma_{33}^{(0)}}{\partial x_{3}}=\frac{\partial \sigma_{3 \beta}^{(1)}}{\partial x_{\beta}}=0
\end{array} ;\left\{\begin{array}{l}
\epsilon_{\alpha \beta}^{(1)}=\frac{1}{2}\left(\frac{\partial u_{\alpha}^{(1)}}{\partial x_{\beta}}+\frac{\partial u_{\beta}^{(1)}}{\partial x_{\alpha}}\right) \\
\epsilon_{\alpha 3}^{(1)}=\frac{1}{2}\left(\frac{\partial u_{\alpha}^{(0)}}{\partial x_{3}}+\frac{\partial u_{3}^{(1)}}{\partial x_{\alpha}}\right)=\frac{1}{2} \frac{\partial u_{3}^{(1)}}{\partial x_{\alpha}} \quad ; \boldsymbol{\epsilon}^{(1)}=\mathbf{C}: \boldsymbol{\sigma}^{(1)} \\
\epsilon_{33}^{(1)}=\frac{\partial u_{3}^{(0)}}{\partial x_{3}}=\frac{\mathrm{d} A_{3}^{(0) 0}}{\mathrm{~d} x_{3}}
\end{array}\right.\right.
$$


where equations (35) have been used, and the cracks are again traction-free. Also, equation (30) yields for $p=k=1$ : $\mathbf{A}^{(0) 1}\left(X_{3}\right)=A_{1}^{(0) 1}\left(X_{3}\right) \mathbf{e}_{1}+A_{2}^{(0) 1}\left(X_{3}\right) \mathbf{e}_{2}=\mathbf{a}^{(1) 1 \pm}\left(x_{3}\right)$, which means (since the expansion of $\mathbf{u}^{(1)}$ cannot contain any term in $x_{2}^{k}$ with $k \geq 2$, see above) that $\mathbf{u}^{(1)}$ must satisfy the condition

$$
\mathbf{u}^{(1)}(\mathbf{x}) \sim\left[A_{1}^{(0) 1}\left(x_{3}\right) \mathbf{e}_{1}+A_{2}^{(0) 1}\left(x_{3}\right) \mathbf{e}_{2}\right] x_{2} \quad \text { for } x_{2} \rightarrow \pm \infty
$$

Again, the problem splits, for every value of $x_{3}$, into two independent problems, for the displacement components $\left(u_{1}^{(1)}, u_{2}^{(1)}\right)$ and $u_{3}^{(1)}$ respectively. The problem for $\left(u_{1}^{(1)}, u_{2}^{(1)}\right)$ is a $2 D$ elasticity problem in generalized plane strain $\left(\epsilon_{33}^{(1)} \neq 0\right.$, independent of $x_{1}$ and $\left.x_{2}\right)$ for a periodic array of inclined cracks subjected to some remote stresses $\sigma_{\alpha \beta}^{(1) \infty}\left(x_{3}\right)$, analogous to that considered in Section 2.

To find the expression of these remote stresses, it suffices to note that by equations (36) and (37), they are exactly identical to those generated in standard 3D elasticity by the displacement field $A_{3}^{(0) 0}\left(X_{3}\right) \mathbf{e}_{3}+\left[A_{1}^{(0) 1}\left(X_{3}\right) \mathbf{e}_{1}+A_{2}^{(0) 1}\left(X_{3}\right) \mathbf{e}_{2}\right] X_{2}$. Now this displacement field is exactly that prevailing near the $\mathrm{CZ}$ in the outer problem at order 0 , see equation (31). It follows that the remote stresses $\sigma_{\alpha \beta}^{(1) \infty}\left(x_{3}\right)$ in the inner problem at order 1 coincide with those on the $C Z$ in the outer problem at order 0:

$$
\sigma_{\alpha \beta}^{(1) \infty}\left(x_{3}\right)=\Sigma_{\alpha \beta}^{(0) \mathrm{CZ}}\left(X_{3}\right) .
$$

On the other hand the problem for $u_{3}^{(1)}$ is a $2 \mathrm{D}$ problem of antiplane elasticity, in which the remote stresses $\sigma_{\alpha 3}^{(1) \infty}$ are zero since $u_{3}^{(1)}$ increases less quickly than $x_{2}$ near infinity, see equation (37) where the right-hand side has no component on the basis vector $\mathbf{e}_{3}$. Thus $u_{3}^{(1)}$ must correspond to a mere rigid-body motion, that is a combination of a translatory motion in the direction $x_{3}$ and rotations about the directions $x_{1}$ and $x_{2}$; but these rotations are impossible, the first because it would imply an asymptotically linear dependence of $u_{3}^{(1)}$ upon $x_{2}$, and the second because it would imply a global rotation about the direction $X_{2}$ in the outer problem. It follows that $u_{3}^{(1)}$ is necessarily of the form

$$
u_{3}^{(1)} \equiv u_{3}^{(1)}\left(x_{3}\right) .
$$

\subsection{Outer problem at order 1}

With regard to the outer problem at order 1, we are not interested in the entire solution but in the sole displacement discontinuity across the $\mathrm{CZ}$, which may be deduced from the matching conditions (30). These conditions yield for $p=1$ and $k=0$ : $\mathbf{A}^{(1) 0 \pm}\left(X_{3}\right)=$ $\mathbf{a}^{(1) 0 \pm}\left(x_{3}\right)$, which implies that the condition

$$
\lim _{X_{2} \rightarrow 0^{ \pm}} \mathbf{U}^{(1)}(\mathbf{X})=\mathbf{a}^{(1) 0 \pm}\left(X_{3}\right)
$$

must be satisfied. It follows, denoting $\llbracket \mathbf{U}^{(1)} \rrbracket$ the discontinuity of $\mathbf{U}^{(1)}$ across the $\mathrm{CZ}$, that

$$
\llbracket \mathbf{U}^{(1)} \rrbracket\left(X_{3}\right)=\mathbf{a}^{(1) 0+}\left(X_{3}\right)-\mathbf{a}^{(1) 0-}\left(X_{3}\right),
$$


which reduces the calculation of $\llbracket \mathbf{U}^{(1)} \rrbracket$ to that of $\mathbf{a}^{(1) 0 \pm}$.

Evaluating $\mathbf{a}^{(1) 0 \pm}$ requires examining the asymptotic behavior of $\mathbf{u}^{(1)}$ near infinity. Consider the components $u_{\alpha}^{(1)}$ first, to which the results of Section 2 are applicable. Let $\left[\mathbf{u}^{(1)}\right] \equiv\left[\mathbf{u}^{(1)}\right]\left(x_{2}, x_{3}\right) \equiv \mathbf{u}^{(1)}\left(x_{2}, x_{3}\right)-\mathbf{u}^{(1)}\left(-x_{2}, x_{3}\right)$ denote the displacement difference between positions located far above and far below the cracks in the inner problem at order 1. By equations (1), (12) $2,3,(3)$ and (38),

$$
\left\{\begin{array}{l}
{\left[u_{1}^{(1)}\right]=q_{3} /(2 \bar{d})=\mathcal{C}_{3 \mu} Q_{\mu} /(2 \bar{d})=\left(\mathcal{C}_{31} \Sigma_{11}^{(0) \mathrm{CZ}}+\mathcal{C}_{32} \Sigma_{22}^{(0) \mathrm{CZ}}+\mathcal{C}_{33} \Sigma_{12}^{(0) \mathrm{CZ}}\right) /(2 \bar{d})} \\
{\left[u_{2}^{(1)}\right]=q_{2} /(2 \bar{d})=\mathcal{C}_{2 \mu} Q_{\mu} /(2 \bar{d})=\left(\mathcal{C}_{21} \Sigma_{11}^{(0) \mathrm{CZ}}+\mathcal{C}_{22} \Sigma_{22}^{(0) \mathrm{CZ}}+\mathcal{C}_{23} \Sigma_{12}^{(0) \mathrm{CZ}}\right) /(2 \bar{d})}
\end{array}\right.
$$

where the $\mathcal{C}_{\lambda \mu}$ denote the compliance coefficients of the rectangle of width $2 \bar{d}$ and height $2 x_{2}$. Now let $f^{\mathrm{NC}}$ denote, for any quantity $f$, its value when no cracks are present and, like in Section 2, $\Delta f$ its variation due to the cracks. Equations (41) may be rewritten as

$$
\left\{\begin{array}{l}
{\left[u_{1}^{(1)}\right]=\left[u_{1}^{(1)}\right]^{\mathrm{NC}}+\Delta\left[u_{1}^{(1)}\right]} \\
{\left[u_{1}^{(1)}\right]^{\mathrm{NC}}=\left(\mathcal{C}_{31}^{\mathrm{NC}} \Sigma_{11}^{(0) \mathrm{CZ}}+\mathcal{C}_{32}^{\mathrm{NC}} \Sigma_{22}^{(0) \mathrm{CZ}}+\mathcal{C}_{33}^{\mathrm{NC}} \Sigma_{12}^{(0) \mathrm{CZ}}\right) /(2 \bar{d})} \\
\Delta\left[u_{1}^{(1)}\right]=\left(\Delta \mathcal{C}_{31} \Sigma_{11}^{(0) \mathrm{CZ}}+\Delta \mathcal{C}_{32} \Sigma_{22}^{(0) \mathrm{CZ}}+\Delta \mathcal{C}_{33} \Sigma_{12}^{(0) \mathrm{CZ}}\right) /(2 \bar{d}) \\
{\left[u_{2}^{(1)}\right]=\left[u_{2}^{(1)}\right]^{\mathrm{NC}}+\Delta\left[u_{2}^{(1)}\right]} \\
{\left[u_{2}^{(1)}\right]^{\mathrm{NC}}=\left(\mathcal{C}_{21}^{\mathrm{NC}} \Sigma_{11}^{(0) \mathrm{CZ}}+\mathcal{C}_{22}^{\mathrm{NC}} \Sigma_{22}^{(0) \mathrm{CZ}}+\mathcal{C}_{23}^{\mathrm{NC}} \Sigma_{12}^{(0) \mathrm{CZ}}\right) /(2 \bar{d})} \\
\Delta\left[u_{2}^{(1)}\right]=\left(\Delta \mathcal{C}_{21} \Sigma_{11}^{(0) \mathrm{CZ}}+\Delta \mathcal{C}_{22} \Sigma_{22}^{(0) \mathrm{CZ}}+\Delta \mathcal{C}_{23} \Sigma_{12}^{(0) \mathrm{CZ}}\right) /(2 \bar{d})
\end{array}\right.
$$

But when no cracks are present, homogeneity of the strain field implies that the displacement differences $\left[u_{\alpha}^{(1)}\right]^{\mathrm{NC}}$ are proportional to $x_{2}$. On the other hand the compliance variations $\Delta \mathcal{C}_{\lambda \mu}$ are independent of $x_{2}$ by equations (17) and (18), so that the same is true of the displacement variations $\Delta\left[u_{\alpha}^{(1)}\right]$. Hence the terms $\left[u_{\alpha}^{(1)}\right]^{\mathrm{NC}}$ and $\Delta\left[u_{\alpha}^{(1)}\right]$ may be identified to the terms proportional to $x_{2}^{1}=x_{2}$ and $x_{2}^{0}=1$ respectively in the expansion of $\left[u_{\alpha}^{(1)}\right]$; that is, for the term proportional to $x_{2}^{0}=1$ :

$$
\left\{\begin{array}{l}
\Delta\left[u_{1}^{(1)}\right]=\left(\Delta \mathcal{C}_{31} \Sigma_{11}^{(0) \mathrm{CZ}}+\Delta \mathcal{C}_{32} \Sigma_{22}^{(0) \mathrm{CZ}}+\Delta \mathcal{C}_{33} \Sigma_{12}^{(0) \mathrm{CZ}}\right) /(2 \bar{d})=a_{1}^{(1) 0+}\left(x_{3}\right)-a_{1}^{(1) 0-}\left(x_{3}\right) \\
\Delta\left[u_{2}^{(1)}\right]=\left(\Delta \mathcal{C}_{21} \Sigma_{11}^{(0) \mathrm{CZ}}+\Delta \mathcal{C}_{22} \Sigma_{22}^{(0) \mathrm{CZ}}+\Delta \mathcal{C}_{23} \Sigma_{12}^{(0) \mathrm{CZ}}\right) /(2 \bar{d})=a_{2}^{(1) 0+}\left(x_{3}\right)-a_{2}^{(1) 0-}\left(x_{3}\right) .
\end{array}\right.
$$

With equation (40), this implies that

$$
\left\{\begin{array}{l}
\llbracket U_{1}^{(1)} \rrbracket=\left(\Delta \mathcal{C}_{31} \Sigma_{11}^{(0) \mathrm{CZ}}+\Delta \mathcal{C}_{32} \Sigma_{22}^{(0) \mathrm{CZ}}+\Delta \mathcal{C}_{33} \Sigma_{12}^{(0) \mathrm{CZ}}\right) /(2 \bar{d}) \\
\llbracket U_{2}^{(1)} \rrbracket=\left(\Delta \mathcal{C}_{21} \Sigma_{11}^{(0) \mathrm{CZ}}+\Delta \mathcal{C}_{22} \Sigma_{22}^{(0) \mathrm{CZ}}+\Delta \mathcal{C}_{23} \Sigma_{12}^{(0) \mathrm{CZ}}\right) /(2 \bar{d})
\end{array}\right.
$$

where the stresses $\Sigma_{\alpha \beta}^{(0) \mathrm{CZ}}$ on the $\mathrm{CZ}$ are given by equations (32). 
For the component $u_{3}^{(1)}$, equation (39) implies that $a_{3}^{(1) 0+}\left(x_{3}\right)=a_{3}^{(1) 0-}\left(x_{3}\right)\left(=u_{3}^{(1)}\left(x_{3}\right)\right)$. It then follows from equation (40) that

$$
\llbracket U_{3}^{(1)} \rrbracket=0
$$

\subsection{Final formulae}

Coming back to the original coordinates $X, Y, Z$, accounting for the fact that the zerothorder displacement field $\mathbf{U}^{(0)}$ is continuous across the $\mathrm{CZ}$ and using equations (17) (with $\bar{d}$ instead of $d),(32),(43)$ and (44), one gets the final expressions looked for of the displacement discontinuities across the $\mathrm{CZ}$ in the outer problem:

$$
\left\{\begin{aligned}
\llbracket U_{X} \rrbracket & =0 \\
\llbracket U_{Y} \rrbracket & =\frac{4\left(1-\nu^{2}\right) d}{E}\left(\mathcal{A}_{22} \Sigma_{Y Y}^{(0) \mathrm{CZ}}+\mathcal{A}_{12} \Sigma_{Z Z}^{(0) \mathrm{CZ}}-\mathcal{A}_{23} \Sigma_{Y Z}^{(0) \mathrm{CZ}}\right) \\
& =\frac{4\left(1-\nu^{2}\right) d}{E \sqrt{2 \pi X}}\left[\left(\mathcal{A}_{22}+2 \nu \mathcal{A}_{12}\right) K_{I}^{(0)}-\mathcal{A}_{23} K_{I I I}^{(0)}\right] \\
\llbracket U_{Z} \rrbracket & =\frac{4\left(1-\nu^{2}\right) d}{E}\left(-\mathcal{A}_{23} \Sigma_{Y Y}^{(0) \mathrm{CZ}}-\mathcal{A}_{13} \Sigma_{Z Z}^{(0) \mathrm{CZ}}+\mathcal{A}_{33} \Sigma_{Y Z}^{(0) \mathrm{CZ}}\right) \\
& =\frac{4\left(1-\nu^{2}\right) d}{E \sqrt{2 \pi X}}\left[-\left(\mathcal{A}_{23}+2 \nu \mathcal{A}_{13}\right) K_{I}^{(0)}+\mathcal{A}_{33} K_{I I I}^{(0)}\right] .
\end{aligned}\right.
$$

\section{The model}

We now come back to the problem depicted in Figure 3. For simplicity, the body is supposed here to be infinite and the initial crack semi-infinite. It is recalled that the remote strain parallel to the direction of the crack front is assumed to be zero. We wish to use the results of the preceding section to schematize, within a macroscopic model, the region of the facets as a $\mathrm{CZ}$.

\subsection{Adaptation of the constitutive law of the cohesive zone}

Directly using, as would seem natural, equations (45) as a constitutive law of the CZ would raise problems near both the initial crack front $(X=0)$ and the tips of the facets $(X=a)$ :

- near the initial crack front because the $1 / \sqrt{X}$ dependence of the stresses $\Sigma_{Y Y}^{(0) \mathrm{CZ}}, \Sigma_{Z Z}^{(0) \mathrm{CZ}}$, $\Sigma_{Y Z}^{(0) \mathrm{CZ}}$ would imply a physically absurd divergence of the displacement discontinuities $\llbracket U_{Y} \rrbracket, \llbracket U_{Z} \rrbracket$ at $X=0$ 
- near the tips of the facets because the same dependence would prevent $\llbracket U_{Y} \rrbracket$, $\llbracket U_{Z} \rrbracket$ from vanishing at $X=a$, as they must.

These difficulties find their origin in shortcomings of the procedure of matched asymptotic expansions used to establish equations (45). Indeed, since this procedure involves dilations of the coordinates in the sole directions $Y$ and $Z$, see equations (21) and (22), it assumes that the variations of the mechanical fields are much quicker in these directions than in the third one, $X$. Now this hypothesis is reasonable over the major part of the facets, because the characteristic distances of variation of the mechanical fields are expected to be of the order of $d$ in the directions $Y$ and $Z$, versus $a(\gg d)$ in the direction $X$. But it clearly breaks down near both the initial crack front and the tips of the facets, where the fully $3 \mathrm{D}$ character of the problem implies that important variations of the mechanical fields must occur over distances of the order of $d$ in the direction $X$.

This analysis however indicates that the constitutive law (45) should be reasonable except in small zones, of typical size $d$, at the origin and end of the CZ. Hence some modification of this law aimed at making its predictions more reasonable at $X=0$ and $X=a$, while leaving it unchanged away from these points, should be acceptable. The proposal made here consists in replacing in a "self-consistent", albeit approximate, manner, the stresses $\Sigma_{Y Y}^{(0) \mathrm{CZ}}, \Sigma_{Z Z}^{(0) \mathrm{CZ}}, \Sigma_{Y Z}^{(0) \mathrm{CZ}}$ at order 0 , that is in the absence of any facets, by the true stresses $\Sigma_{Y Y}^{\mathrm{CZ}}, \Sigma_{Z Z}^{\mathrm{CZ}}, \Sigma_{Y Z}^{\mathrm{CZ}}$ when these facets are present; equations (45) then become

$$
\left\{\begin{array}{l}
\llbracket U_{X} \rrbracket=0 \\
\llbracket U_{Y} \rrbracket=\frac{4\left(1-\nu^{2}\right) d}{E}\left(\mathcal{A}_{22} \Sigma_{Y Y}^{\mathrm{CZ}}+\mathcal{A}_{12} \Sigma_{Z Z}^{\mathrm{CZ}}-\mathcal{A}_{23} \Sigma_{Y Z}^{\mathrm{CZ}}\right) \\
\llbracket U_{Z} \rrbracket=\frac{4\left(1-\nu^{2}\right) d}{E}\left(-\mathcal{A}_{23} \Sigma_{Y Y}^{\mathrm{CZ}}-\mathcal{A}_{13} \Sigma_{Z Z}^{\mathrm{CZ}}+\mathcal{A}_{33} \Sigma_{Y Z}^{\mathrm{CZ}}\right) .
\end{array}\right.
$$

It may be anticipated, and will be fully confirmed below, that away from the points $X=0$ and $X=a$, the true stresses on the $\mathrm{CZ}$ will differ little from their counterparts at order 0 , so that equations (46) will be essentially equivalent to (45). On the other hand, in small boundary layers of thickness proportional to $d$ at the endpoints of the $\mathrm{CZ}$, the true stresses will adjust so as to fulfill the conditions of finiteness and vanishing of the displacement discontinuities at $X=0$ and $X=a$, respectively.

\subsection{Integral equations}

The region of the facets being approximately represented by a CZ obeying the constitutive law (46), the situation schematized in Figure 4(a) may be considered as a linear superposition of the two situations depicted in Figure 5:

- Situation (A) where the far loading is imposed in the absence of facets, that is, the CZ remaining closed (Figure 5(a); this exactly corresponds, in the matched asymptotic expansion procedure of Section 3, to the outer problem at order 0$)$. The loading generates the $\mathrm{SIF} K_{I}^{(0)}, K_{I I I}^{(0)}$ on the initial crack front $O Z$. The stresses $\Sigma_{Y Y}^{(\mathrm{A}) \mathrm{CZ}}, \Sigma_{X X}^{(\mathrm{A}) \mathrm{CZ}}$, 
$\Sigma_{Z Z}^{(\mathrm{A}) \mathrm{CZ}}, \Sigma_{Y Z}^{(\mathrm{A}) \mathrm{CZ}}$ on the $\mathrm{CZ}$ are given by formulae similar to (32), and the displacement discontinuity $\llbracket \mathbf{U}^{(\mathrm{A})} \rrbracket$ across this $\mathrm{CZ}$ is zero.

- Situation (B) where no far loading is imposed but the upper $(+)$ and lower $(-)$ faces of the $\mathrm{CZ}$ are opened by surface tractions $\pm\left[p(X) \mathbf{e}_{Y}+q(X) \mathbf{e}_{Z}\right]$, where $p(X)$ and $q(X)$ denote unknown functions and $\mathbf{e}_{Y}$ and $\mathbf{e}_{Z}$ the unit vectors parallel to the directions $Y$ and $Z$ (Figure 5(b)). The solution of this problem is classical (for a semi-infinite crack in an infinite body and a zero strain in the direction of the crack front, as assumed in this section). The stresses on the $\mathrm{CZ}$ are given by

$$
\begin{cases}\Sigma_{Y Y}^{(\mathrm{B}) \mathrm{CZ}}=\Sigma_{X X}^{(\mathrm{B}) \mathrm{CZ}} & =-p(X) \\ \Sigma_{Z Z}^{(\mathrm{B}) \mathrm{CZ}}=\nu\left(\Sigma_{X X}^{(\mathrm{B}) \mathrm{CZ}}+\Sigma_{Y Y}^{(\mathrm{B}) \mathrm{CZ}}\right) & =-2 \nu p(X) \\ \Sigma_{Y Z}^{(\mathrm{B}) \mathrm{CZ}} & =-q(X)\end{cases}
$$

and the displacement discontinuities across the CZ obey the classical LEFM equations

$$
\left\{\begin{array}{l}
\frac{\mathrm{d} \llbracket U_{Y}^{(B)} \rrbracket}{\mathrm{d} X}=\frac{4\left(1-\nu^{2}\right)}{\pi E} P V \int_{0}^{a} p\left(X^{\prime}\right) \sqrt{\frac{a-X^{\prime}}{a-X}} \frac{\mathrm{d} X^{\prime}}{X^{\prime}-X} \\
\frac{\mathrm{d} \llbracket U_{Z}^{(B)} \rrbracket}{\mathrm{d} X}=\frac{4(1+\nu)}{\pi E} P V \int_{0}^{a} q\left(X^{\prime}\right) \sqrt{\frac{a-X^{\prime}}{a-X}} \frac{\mathrm{d} X^{\prime}}{X^{\prime}-X}
\end{array}\right.
$$

where the symbol $P V$ denotes a Cauchy principal value.

In the resulting situation $(\mathrm{A})+(\mathrm{B})$, the stresses $\Sigma_{i j}^{\mathrm{CZ}}=\Sigma_{i j}^{(\mathrm{A}) \mathrm{CZ}}+\Sigma_{i j}^{(\mathrm{B}) \mathrm{CZ}}$ on the $\mathrm{CZ}$ are given by

$$
\begin{cases}\Sigma_{Y Y}^{\mathrm{CZ}}=\Sigma_{X X}^{\mathrm{CZ}} & =\frac{K_{I}^{(0)}}{\sqrt{2 \pi X}}-p(X) \\ \Sigma_{Z Z}^{\mathrm{CZ}}=\nu\left(\Sigma_{X X}^{\mathrm{CZ}}+\Sigma_{Y Y}^{\mathrm{CZ}}\right) & =2 \nu\left(\frac{K_{I}^{(0)}}{\sqrt{2 \pi X}}-p(X)\right) \\ \Sigma_{Y Z}^{\mathrm{CZ}} & =\frac{K_{I I I}^{(0)}}{\sqrt{2 \pi X}}-q(X)\end{cases}
$$

and the displacement discontinuities $\llbracket U_{i} \rrbracket=\llbracket U_{i}^{(A)} \rrbracket+\llbracket U_{i}^{(B)} \rrbracket$ across the CZ obey the same equations as in situation $(\mathrm{B})$ :

$$
\left\{\begin{array}{l}
\frac{\mathrm{d} \llbracket U_{Y} \rrbracket}{\mathrm{d} X}=\frac{4\left(1-\nu^{2}\right)}{\pi E} P V \int_{0}^{a} p\left(X^{\prime}\right) \sqrt{\frac{a-X^{\prime}}{a-X}} \frac{\mathrm{d} X^{\prime}}{X^{\prime}-X} \\
\frac{\mathrm{d} \llbracket U_{Z} \rrbracket}{\mathrm{d} X}=\frac{4(1+\nu)}{\pi E} P V \int_{0}^{a} q\left(X^{\prime}\right) \sqrt{\frac{a-X^{\prime}}{a-X}} \frac{\mathrm{d} X^{\prime}}{X^{\prime}-X} .
\end{array}\right.
$$

Also, these discontinuities are given by the constitutive law (46), with $\Sigma_{Y Y}^{\mathrm{CZ}}, \Sigma_{Z Z}^{\mathrm{CZ}}, \Sigma_{Y Z}^{\mathrm{CZ}}$ given by equations (47). Differentiating the expressions of $\llbracket U_{Y} \rrbracket$ and $\llbracket U_{Z} \rrbracket$ with respect to $X$ and eliminating the derivatives $\mathrm{d} \llbracket U_{Y} \rrbracket / \mathrm{d} X$ and $\mathrm{d} \llbracket U_{Z} \rrbracket / \mathrm{d} X$ between the expressions found and equations (48), one finds the following 1D integral equations on the unknown 

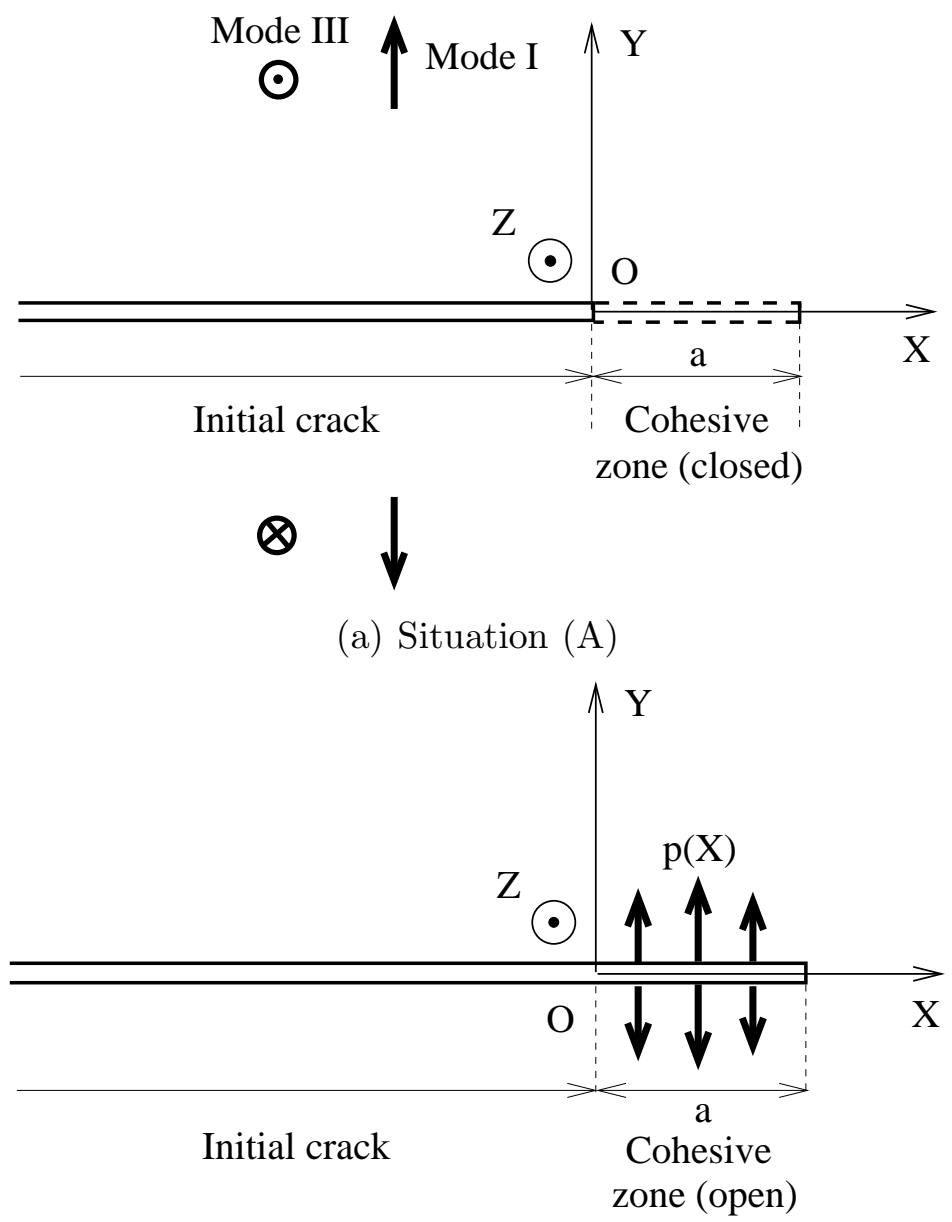

(b) Situation (B) (the sole tractions $\pm p(X) \mathbf{e}_{Y}$ are represented for legibility)

Fig. 5. Linear decomposition of the situation of Figure 4(a) into two situations

tractions $p(X)$ and $q(X)$ :

$$
\left\{\begin{array}{c}
\left(\mathcal{A}_{22}+2 \nu \mathcal{A}_{12}\right) \frac{\mathrm{d} p}{\mathrm{~d} X}-\mathcal{A}_{23} \frac{\mathrm{d} q}{\mathrm{~d} X}+\frac{1}{\pi d} P V \int_{0}^{a} p\left(X^{\prime}\right) \sqrt{\frac{a-X^{\prime}}{a-X}} \frac{\mathrm{d} X^{\prime}}{X^{\prime}-X} \\
=\frac{-\left(\mathcal{A}_{22}+2 \nu \mathcal{A}_{12}\right) K_{I}^{(0)}+\mathcal{A}_{23} K_{I I I}^{(0)}}{2 \sqrt{2 \pi} X^{3 / 2}} \\
-\left(\mathcal{A}_{23}+2 \nu \mathcal{A}_{13}\right) \frac{\mathrm{d} p}{\mathrm{~d} X}+\mathcal{A}_{33} \frac{\mathrm{d} q}{\mathrm{~d} X}+\frac{1}{\pi(1-\nu) d} P V \int_{0}^{a} q\left(X^{\prime}\right) \sqrt{\frac{a-X^{\prime}}{a-X}} \frac{\mathrm{d} X^{\prime}}{X^{\prime}-X} \\
=\frac{\left(\mathcal{A}_{23}+2 \nu \mathcal{A}_{13}\right) K_{I}^{(0)}-\mathcal{A}_{33} K_{I I I}^{(0)}}{2 \sqrt{2 \pi} X^{3 / 2}} .
\end{array}\right.
$$

These integral equations must be supplemented with boundary conditions resulting from the fact that the displacement discontinuities are necessarily zero at the endpoint of the 
$\mathrm{CZ}(X=a)$. Defining the quantities

$$
\left\{\begin{array}{l}
\mathcal{X} \equiv \frac{K_{I}^{(0)}}{\sqrt{2 \pi a}}-p(a) \\
\mathcal{Y} \equiv \frac{K_{I I I}^{(0)}}{\sqrt{2 \pi a}}-q(a)
\end{array}\right.
$$

one gets from equations (46) and (47)

$$
\left\{\begin{array}{l}
\left(\mathcal{A}_{22}+2 \nu \mathcal{A}_{12}\right) \mathcal{X}-\mathcal{A}_{23} \mathcal{Y}=0 \\
-\left(\mathcal{A}_{23}+2 \nu \mathcal{A}_{13}\right) \mathcal{X}+\mathcal{A}_{33} \mathcal{Y}=0
\end{array}\right.
$$

But the coefficients $\mathcal{A}_{\lambda \mu}$ are not tied by any special relation other than those resulting from symmetry; hence the determinant of this linear system in $(\mathcal{X}, \mathcal{Y})$ is nonzero. Therefore it admits only the trivial solution $(\mathcal{X}, \mathcal{Y})=(0,0)$, which means that

$$
\left\{\begin{array}{l}
p(a)=\frac{K_{I}^{(0)}}{\sqrt{2 \pi a}} \\
q(a)=\frac{K_{I I I}^{(0)}}{\sqrt{2 \pi a}}
\end{array}\right.
$$

Once the solution $(p, q)$ of equations $(49,50)$ is known, one may calculate the "global SIF" $K_{I}, K_{I I I}$ along the line $X=a$ connecting the tips of the facets, from which readily follows, through Irwin's formula, the "global energy-release-rate" $G$ due to propagation of these facets. Indeed these SIF are zero in situation (A) in which the CZ is closed, so that they arise only from the surface tractions $\pm\left[p(X) \mathbf{e}_{Y}+q(X) \mathbf{e}_{Z}\right]$ exerted onto the faces of the $\mathrm{CZ}$ in situation $(\mathrm{B})$, and are therefore given by the classical formulae

$$
\left\{\begin{array}{l}
K_{I}=\sqrt{\frac{2}{\pi}} \int_{0}^{a} p(X) \frac{\mathrm{d} X}{\sqrt{a-X}} \\
K_{I I I}=\sqrt{\frac{2}{\pi}} \int_{0}^{a} q(X) \frac{\mathrm{d} X}{\sqrt{a-X}} .
\end{array}\right.
$$

\subsection{Variant incorporating facet coarsening}

In the derivation of equations (49), all geometric parameters $c, d, \alpha$ of the facets were implicitly assumed to be independent of the position $X$ along these facets. While this hypothesis is reasonable for the tilt angle $\alpha$ and the ratio $c / d$ of the projected facet width to the geometrical period, it is more disputable for $c$ and $d$ individually because of the currently observed coarsening of facets. This phenomenon is illustrated in Figure 6, which shows a recent photograph of the plexiglas specimens described in (Lazarus et al., 2008); in this picture the initial crack is located at the very top and propagates toward the bottom. Although the geometrical period (in the horizontal direction) is much smaller than the facet length (in the vertical direction), as assumed in the theoretical 
analysis, this period does not remain constant as the facets propagate because they tend to gradually coalesce. At least three or four coalescence periods are observable in the picture, and in all probability would have been followed by other ones had propagation gone on. Facet coarsening was also conspicuous in Pons and Karma (2010)'s phase-field numerical simulations, see Figures 1(c) and 1(d) of their paper.

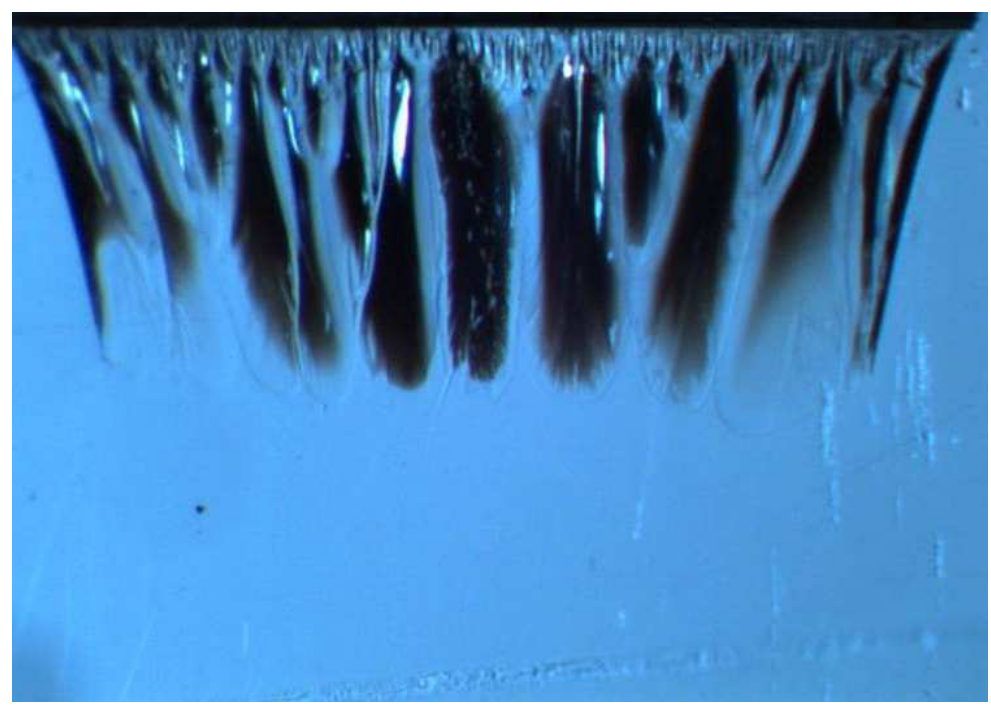

Fig. 6. Fracture facets in mode I+III - Experiment by Buchholz, photograph by Lazarus

Disregarding the geometrical complexities of the fracture surface in the coalescence regions, one may propose a simple CZ model including facet coarsening by merely introducing a dependence of $c$ and $d$ upon $X$ in the preceding approach (both $c / d$ and $\alpha$ being assumed to remain independent of $X$ ). Equations (46), (47) and (48) still apply then without any change, but one must account for the dependence of $d$ upon $X$ when differentiating the expressions (46) of the displacement discontinuities $\llbracket U_{Y} \rrbracket, \llbracket U_{Z} \rrbracket$. Doing so, one obtains the following slightly more complex integral equations:

$$
\left\{\begin{array}{c}
\left(\mathcal{A}_{22}+2 \nu \mathcal{A}_{12}\right)\left[\frac{\mathrm{d} p}{\mathrm{~d} X}+\frac{\mathrm{d}(\ln d)}{\mathrm{d} X} p\right]-\mathcal{A}_{23}\left[\frac{\mathrm{d} q}{\mathrm{~d} X}+\frac{\mathrm{d}(\ln d)}{\mathrm{d} X} q\right] \\
+\frac{1}{\pi d} P V \int_{0}^{a} p\left(X^{\prime}\right) \sqrt{\frac{a-X^{\prime}}{a-X}} \frac{\mathrm{d} X^{\prime}}{X^{\prime}-X} \\
=\frac{-\left(\mathcal{A}_{22}+2 \nu \mathcal{A}_{12}\right) K_{I}^{(0)}+\mathcal{A}_{23} K_{I I I}^{(0)}}{\sqrt{2 \pi}}\left[\frac{1}{2 X^{3 / 2}}-\frac{1}{\sqrt{X}} \frac{\mathrm{d}(\ln d)}{\mathrm{d} X}\right] \\
-\left(\mathcal{A}_{23}+2 \nu \mathcal{A}_{13}\right)\left[\frac{\mathrm{d} p}{\mathrm{~d} X}+\frac{\mathrm{d}(\ln d)}{\mathrm{d} X} p\right]+\mathcal{A}_{33}\left[\frac{\mathrm{d} q}{\mathrm{~d} X}+\frac{\mathrm{d}(\ln d)}{\mathrm{d} X} q\right] \\
+\frac{1}{\pi(1-\nu) d} P V \int_{0}^{a} q\left(X^{\prime}\right) \sqrt{\frac{a-X^{\prime}}{a-X^{\prime}}} \frac{\mathrm{d} X^{\prime}}{X^{\prime}-X} \\
=\frac{\left(\mathcal{A}_{23}+2 \nu \mathcal{A}_{13}\right) K_{I}^{(0)}-\mathcal{A}_{33} K_{I I I}^{(0)}}{\sqrt{2 \pi}}\left[\frac{1}{2 X^{3 / 2}}-\frac{1}{\sqrt{X}} \frac{\mathrm{d}(\ln d)}{\mathrm{d} X}\right] .
\end{array}\right.
$$




\subsection{Numerical method of solution}

We come back to the simpler version (49) of the integral equations disregarding facet coarsening, leaving exploitation of the more complete ones (52) for future work. Since these equations do not seem to be amenable to some analytic solution, a strategy for a numerical solution must be developed.

A preliminary step consists in putting the equations in a more convenient form by introducing suitable changes of variables and functions. First, one may use dimensionless coordinates $w, w^{\prime}$ defined by

$$
w \equiv \frac{X}{a} \quad ; \quad w^{\prime} \equiv \frac{X^{\prime}}{a} .
$$

Second, one may guess from equations (49), disregarding the integral terms, that the unknown tractions $p, q$ probably diverge like $X^{-1 / 2}$ in the limit $X \rightarrow 0$; then, postulating such a singular behavior, one may check that it is indeed correct because the integral terms then diverge only like $X^{-1 / 2}$, versus $X^{-3 / 2}$ for the right-hand sides. ${ }^{5}$ This suggests to use, instead of $p$ and $q$, the functions $\bar{p}$ and $\bar{q}$ defined by

$$
p(X) \equiv \frac{\bar{p}(w)}{\sqrt{w}} \quad ; \quad q(X) \equiv \frac{\bar{q}(w)}{\sqrt{w}},
$$

so as to work with functions having finite limits for $X \rightarrow 0$. With these new coordinates and functions, the integral equations (49) take the form

$$
\left\{\begin{array}{l}
\left(\mathcal{A}_{22}+2 \nu \mathcal{A}_{12}\right)\left(w \frac{\mathrm{d} \bar{p}}{\mathrm{~d} w}-\frac{\bar{p}}{2}\right)-\mathcal{A}_{23}\left(w \frac{\mathrm{d} \bar{q}}{\mathrm{~d} w}-\frac{\bar{q}}{2}\right) \\
+\frac{a / d}{\pi} \frac{w^{3 / 2}}{\sqrt{1-w}} P V \int_{0}^{1} \bar{p}\left(w^{\prime}\right) \sqrt{\frac{1-w^{\prime}}{w^{\prime}}} \frac{\mathrm{d} w^{\prime}}{w^{\prime}-w}=\frac{-\left(\mathcal{A}_{22}+2 \nu \mathcal{A}_{12}\right) K_{I}^{(0)}+\mathcal{A}_{23} K_{I I I}^{(0)}}{2 \sqrt{2 \pi a}} \\
-\left(\mathcal{A}_{23}+2 \nu \mathcal{A}_{13}\right)\left(w \frac{\mathrm{d} \bar{p}}{\mathrm{~d} w}-\frac{\bar{p}}{2}\right)+\mathcal{A}_{33}\left(w \frac{\mathrm{d} \bar{q}}{\mathrm{~d} w}-\frac{\bar{q}}{2}\right) \\
+\frac{a / d}{\pi(1-\nu)} \frac{w^{3 / 2}}{\sqrt{1-w}} P V \int_{0}^{1} \bar{q}\left(w^{\prime}\right) \sqrt{\frac{1-w^{\prime}}{w^{\prime}}} \frac{\mathrm{d} w^{\prime}}{w^{\prime}-w}=\frac{\left(\mathcal{A}_{23}+2 \nu \mathcal{A}_{13}\right) K_{I}^{(0)}-\mathcal{A}_{33} K_{I I I}^{(0)}}{2 \sqrt{2 \pi a}} .
\end{array}\right.
$$

Now introduce a set of $N+1$ nodes $0=w_{0}^{\prime}<w_{1}^{\prime}<\ldots<w_{N-1}^{\prime}<w_{N}^{\prime}=1$ and a set of $N$ collocation points $w_{1}<w_{2}<\ldots<w_{N}$ located at the midpoints of the intervals $\left[w_{i-1}^{\prime}, w_{i}^{\prime}\right]$ :

$$
w_{i} \equiv \frac{1}{2}\left(w_{i-1}^{\prime}+w_{i}^{\prime}\right) \quad(i=1,2, \ldots, N),
$$

and denote $\bar{p}_{0}, \bar{p}_{1}, \ldots, \bar{p}_{N}, \bar{q}_{0}, \bar{q}_{1}, \ldots, \bar{q}_{N}$ the nodal values of the functions $\bar{p}, \bar{q}$ :

$$
\bar{p}_{i} \equiv \bar{p}\left(w_{i}^{\prime}\right) \quad ; \quad \bar{q}_{i} \equiv \bar{q}\left(w_{i}^{\prime}\right) \quad(i=0,1, \ldots, N) .
$$

5 The $X^{-1 / 2}$ behavior of $p$ and $q$ is also necessary in view of the finiteness of $\llbracket U_{Y} \rrbracket$ and $\llbracket U_{Z} \rrbracket$ at $X=0$ which implies, via equations (46) and (47), that $K_{I}^{(0)} / \sqrt{2 \pi X}-p$ and $K_{I I I}^{(0)} / \sqrt{2 \pi X}-q$ must remain bounded in the limit $X \rightarrow 0$. 
Then write equations (49) at the collocation points, by

- replacing the exact values of $\bar{p}$ and $\mathrm{d} \bar{p} / \mathrm{d} w$ at the point $w_{i}$ by the approximate ones $\frac{1}{2}\left(\bar{p}_{i-1}+\bar{p}_{i}\right)$ and $\left(\bar{p}_{i}-\bar{p}_{i-1}\right) /\left(w_{i}^{\prime}-w_{i-1}^{\prime}\right)$ respectively, and similarly for $\bar{q}$;

- using linear interpolations of $\bar{p}$ and $\bar{q}$ in each interval $\left[w_{j-1}^{\prime}, w_{j}^{\prime}\right]$ to calculate the integrals.

The following equations are obtained:

$$
\left\{\begin{array}{c}
\left(\mathcal{A}_{22}+2 \nu \mathcal{A}_{12}\right)\left[w_{i} \frac{\bar{p}_{i}-\bar{p}_{i-1}}{w_{i}^{\prime}-w_{i-1}^{\prime}}-\frac{1}{4}\left(\bar{p}_{i-1}+\bar{p}_{i}\right)\right]-\mathcal{A}_{23}\left[w_{i} \frac{\bar{q}_{i}-\bar{q}_{i-1}}{w_{i}^{\prime}-w_{i-1}^{\prime}}-\frac{1}{4}\left(\bar{q}_{i-1}+\bar{q}_{i}\right)\right] \\
+\frac{a / d}{\pi} \frac{w_{i}^{3 / 2}}{\sqrt{1-w_{i}}} \sum_{j=1}^{N}\left(M_{i j} \frac{w_{j}^{\prime} \bar{p}_{j-1}-w_{j-1}^{\prime} \bar{p}_{j}}{w_{j}^{\prime}-w_{j-1}^{\prime}}+N_{i j} \frac{\bar{p}_{j}-\bar{p}_{j-1}}{w_{j}^{\prime}-w_{j-1}^{\prime}}\right) \\
=\frac{-\left(\mathcal{A}_{22}+2 \nu \mathcal{A}_{12}\right) K_{I}^{(0)}+\mathcal{A}_{23} K_{I I I}^{(0)}}{2 \sqrt{2 \pi a}} \quad(i=1,2, \ldots, N) \\
-\left(\mathcal{A}_{23}+2 \nu \mathcal{A}_{13}\right)\left[w_{i} \frac{\bar{p}_{i}-\bar{p}_{i-1}}{w_{i}^{\prime}-w_{i-1}^{\prime}}-\frac{1}{4}\left(\bar{p}_{i-1}+\bar{p}_{i}\right)\right]+\mathcal{A}_{33}\left[w_{i} \frac{\bar{q}_{i}-\bar{q}_{i-1}}{w_{i}^{\prime}-w_{i-1}^{\prime}}-\frac{1}{4}\left(\bar{q}_{i-1}+\bar{q}_{i}\right)\right] \\
+\frac{\left.w_{j}^{\prime}\right)}{\pi(1-\nu)} \frac{w_{i}^{3 / 2}}{\sqrt{1-w_{i}}} \sum_{j=1}^{N}\left(M_{i j} \frac{w_{j}^{\prime} \bar{q}_{j-1}-w_{j-1}^{\prime} \bar{q}_{j}}{w_{j}^{\prime}-w_{j-1}^{\prime}}+N_{i j} \frac{\bar{q}_{j}-\bar{q}_{j-1}}{w_{j}^{\prime}-w_{j-1}^{\prime}}\right) \\
=\frac{\left(\mathcal{A}_{23}+2 \nu \mathcal{A}_{13}\right) K_{I}^{(0)}-\mathcal{A}_{33} K_{I I I}^{(0)}}{2 \sqrt{2 \pi a}} \quad(i=1,2, \ldots, N)
\end{array}\right.
$$

where

$$
\left\{\begin{array}{l}
M_{i j} \equiv P V \int_{w_{j-1}^{\prime}}^{w_{j}^{\prime}} \sqrt{\frac{1-w^{\prime}}{w^{\prime}}} \frac{\mathrm{d} w^{\prime}}{w^{\prime}-w_{i}} \\
N_{i j} \equiv P V \int_{w_{j-1}^{\prime}}^{w_{j}^{\prime}} \sqrt{w^{\prime}\left(1-w^{\prime}\right)} \frac{\mathrm{d} w^{\prime}}{w^{\prime}-w_{i}}
\end{array}\right.
$$

these integrals are calculable analytically, see their expressions in Appendix C. The discretized integral equations (58) are completed by the boundary conditions resulting from equations (50):

$$
\left\{\begin{array}{l}
\bar{p}(w=1)=\bar{p}_{N}=\frac{K_{I}^{(0)}}{\sqrt{2 \pi a}} \\
\bar{q}(w=1)=\bar{q}_{N}=\frac{K_{I I I}^{(0)}}{\sqrt{2 \pi a}} .
\end{array}\right.
$$

Equations (58) and (60) together form a system of $2 N+2$ linear equations on the $2 N+2$ unknowns $\bar{p}_{0}, \bar{p}_{1}, \ldots, \bar{p}_{N}, \bar{q}_{0}, \bar{q}_{1}, \ldots, \bar{q}_{N}$. This system may be solved numerically; the matrix implied is of course full but of reasonable size provided that no more than a few hundred nodes are used, which is quite sufficient in practice. No time-consuming numerical calculations of integrals are required since the coefficients $M_{i j}$ and $N_{i j}$ are known in analytic form, see Appendix C.

Once the system $(58,60)$ is solved, the global SIF may be obtained from the discretized 
form of equations (51):

$$
\left\{\begin{array}{l}
K_{I}=\sqrt{\frac{2 a}{\pi}} \sum_{j=1}^{N} \int_{w_{j-1}^{\prime}}^{w_{j}^{\prime}} \bar{p}(w) \frac{\mathrm{d} w}{\sqrt{w(1-w)}} \\
K_{I I I}=\sqrt{\frac{2 a}{\pi}} \sum_{j=1}^{N} \int_{w_{j-1}^{\prime}}^{w_{j}^{\prime}} \bar{q}(w) \frac{\mathrm{d} w}{\sqrt{w(1-w)}}
\end{array}\right.
$$

the calculation of the integrals here is elementary using a linear interpolation of the functions $\bar{p}, \bar{q}$ in each interval $\left[w_{j-1}^{\prime}, w_{j}^{\prime}\right]$ and the change of variable $w=\sin ^{2} \theta$.

\subsection{Examples}

Complete exploitation of the model based on the numerical algorithm just expounded is left for future work; we shall be content here with a few numerical illustrations concentrating essentially on the influence of the parameter $d / a$. All these illustrations use the approximate values of the coefficients $\mathcal{A}_{\lambda \mu}$ provided in Appendix B, but again substituting more accurate ones would not raise any problem other than their present lack of existence.

The domain of study $0 \leq w \leq 1(0 \leq X \leq a)$ is discretized using 51 nodes and 50 collocation points. (These numbers are large enough to warrant mesh independence). The mesh is refined near the endpoints $w=0$ and $w=1$ since boundary layers are expected there, see Subsection 4.1. The parameters used are $\nu=0.3, K_{I I I}^{(0)} / K_{I}^{(0)}=0.15, c / d=0.6, \alpha=18.4^{\circ}$, and the value of $d / a$ is varied between 0.01 and 0.5 . These values are not arbitrary (except for those of the varying parameter $d / a)$ : those of $\nu, K_{I I I}^{(0)} / K_{I}^{(0)}, c / d$ correspond to an actual experiment performed by Lazarus et al. (2008); and that of $\alpha$ derives from Pollard et al. (1982)'s predictive formula for the tilt angle, $\alpha=\frac{1}{2} \arctan \left[K_{I I I}^{(0)} /\left(\left(\frac{1}{2}-\nu\right) K_{I}^{(0)}\right)\right]$, resulting from the heuristic hypothesis that facets develop along the plane which, for the initial stress field prior to their propagation, is free of shear tractions.

Figure 7 shows the functions $\bar{p}(w), \bar{q}(w)$ obtained, normalized by the value $K_{I}^{(0)} / \sqrt{2 \pi a}$ of $\bar{p}$ at $w=1$. Several points are noteworthy here:

- The functions $\bar{p}(w)$ and $\bar{q}(w)$ remain finite at $w=0$, as was theoretically predicted in Subsection 4.4.

- When the ratio $d / a$ goes to zero, in the major part of the interval $[0,1], \bar{p}(w)$ and $\bar{q}(w)$ also go to zero so that the true stresses $\Sigma_{Y Y}^{\mathrm{CZ}}, \Sigma_{Z Z}^{\mathrm{CZ}}, \Sigma_{Y Z}^{\mathrm{CZ}}$ on the CZ go to their counterparts $\Sigma_{Y Y}^{(0) \mathrm{CZ}}, \Sigma_{Z Z}^{(0) \mathrm{CZ}}, \Sigma_{Y Z}^{(0) \mathrm{CZ}}$ at order 0; again, this agrees with what was anticipated in Subsection 4.1.

- However boundary layers where $\bar{p}(w)$ and $\bar{q}(w)$ vary quickly, of size decreasing with $d / a$, may be observed at the endpoints of the interval $[0,1]$, once again in agreement with what was foreseen in Subsection 4.1.

It is interesting to note that elementary asymptotic studies of the integral equations (49) in the limit of very small values of the ratio $d / a$ support these numerical findings: 


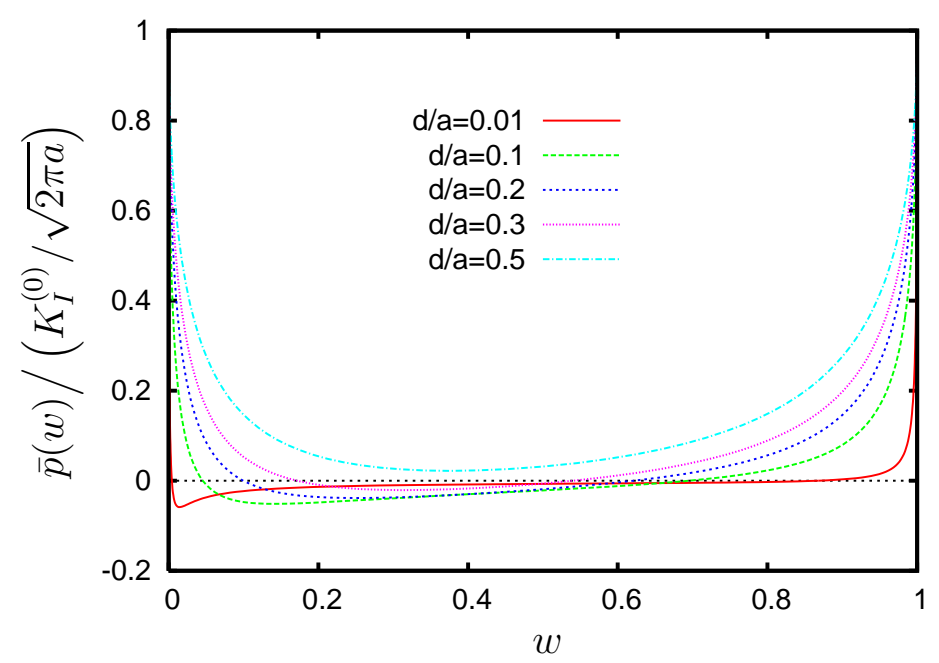

(a) The function $\bar{p}(w)$

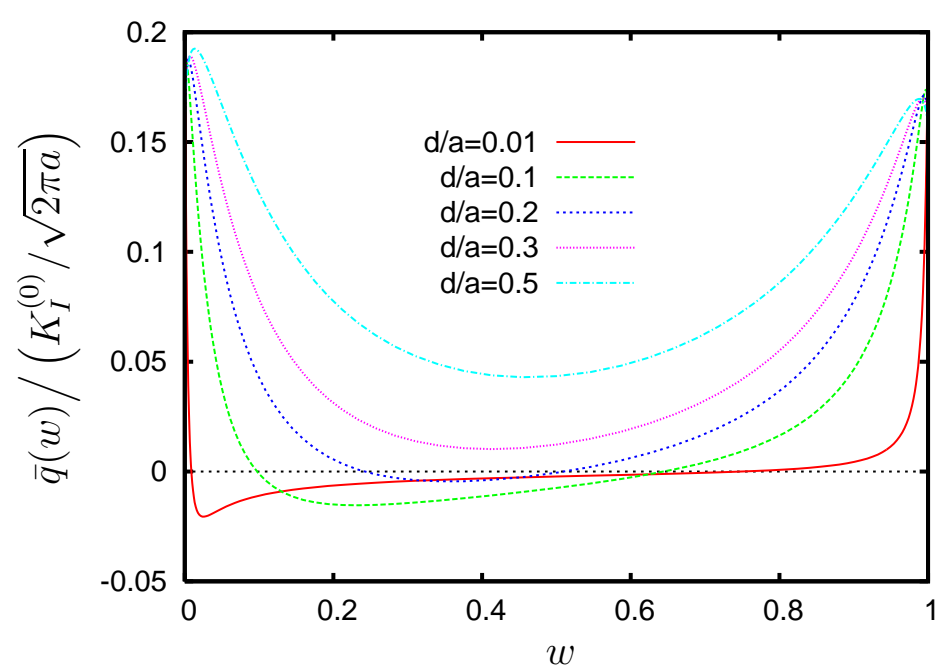

(b) The function $\bar{q}(w)$

Fig. 7. The functions $\bar{p}(w)$ and $\bar{q}(w)$ for $\nu=0.3, K_{I I I}^{(0)} / K_{I}^{(0)}=0.15, c / d=0.6, \alpha=18.4^{\circ}$ and various values of $d / a$

- The behavior of the solution away from the endpoints of the CZ may be studied by fixing $X, X \neq 0, X \neq a$, while letting $d / a$ go to 0 . It is easily found that $p(X)$ and $q(X)$ are of order $O(d / a)$. This confirms that away from the endpoints, the difference between the stresses on the $\mathrm{CZ}$ and their counterparts at order 0 goes to 0 with $d / a$.

- The vicinity of the left endpoint $X=0$ may be studied by fixing $X / d$ (instead of $X$ ) while letting $d / a$ go to 0 . Assuming the initial SIF $K_{I}^{(0)}, K_{I I I}^{(0)}$ to be of comparable magnitudes, it is found that $p(X), q(X)$ are asymptotically of the form $\left(K_{I \text { or } I I I} / \sqrt{d}\right) f_{L}(X / d)$, $\left(K_{I \text { or } I I I} / \sqrt{d}\right) g_{L}(X / d)$ where $f_{L}, g_{L}$ are dimensionless functions and the subscripts $\mathrm{L}$ refers to the left endpoint. This confirms existence of a boundary layer near the point $X=0$, of thickness proportional to $d$.

- Finally the vicinity of the right endpoint $X=a$ may be studied by fixing $(a-X) / d$ while letting $d / a$ go to 0 , with the conclusion that $p(X), q(X)$ are asymptotically of the form $\left(K_{I \text { or } I I I} / \sqrt{a}\right) f_{R}((a-X) / d),\left(K_{I \text { or } I I I} / \sqrt{a}\right) g_{R}((a-X) / d)$ where $f_{R}, g_{R}$ are dimensionless functions and the subscripts $R$ refers to the right endpoint. This confirms 
the existence of another boundary layer of thickness of order $d{ }^{6}$

Coming back to numerical results, Figure 8 shows the ratio, deduced from equations (61) with the functions $\bar{p}(w), \bar{q}(w)$ determined numerically, of the global energy-release-rate $G$ to the initial energy-release rate $G^{(0)}$ prior to propagation of the facets. Again, the parameters used are $\nu=0.3, K_{I I I}^{(0)} / K_{I}^{(0)}=0.15$ and $\alpha=18.4^{\circ}$; in Figure $8(\mathrm{a}), c / d=0.6$ and the value of $d / a$ varies between from 0 to 1 , whereas in Figure 8(b), $d / a=0.3$ and $c / d$ varies between 0 and 1 . Again, several points are noteworthy:

- In all cases $G / G^{(0)}$ is smaller than unity, which means that the body releases less elastic energy through propagation of tilted facets than through propagation of a planar crack. This is because in the case of tilted facets the release of elastic energy is hindered by the permanence of unbroken ligaments between them. Note that although this conclusion is based on results obtained using Leblond and Frelat (2014)'s approximate solution, applicable to the sole case where $c<d$, it is by no means limited to this case. Indeed it is intuitively obvious, and confirmed by Fleck (1991)'s numerical calculations (see his Figure 5) that as soon as $\alpha$ is nonzero, even if $c>d$, small unbroken ligaments persist and still hamper the release of elastic energy.

- The ratio $G / G^{(0)}$ goes down to zero when the ratio $d / a$ goes to zero, the ratio $c / d$ remaining fixed. This is because according to equations (46), a decrease of the geometrical period for fixed facet length induces a decrease of the displacement discontinuities across the $\mathrm{CZ}$, that is a closure of this $\mathrm{CZ}$ unfavorable to the release of elastic energy.

- The ratio $G / G^{(0)}$ is an increasing function of $c / d$ for fixed $d / a$. The increase is very quick when $c / d$ goes to unity, because in this limit the unbroken ligaments preventing the release of elastic energy shrink rapidly. But the use of Leblond and Frelat (2014)'s approximate solution certainly leads here to some overestimation of the effect, since as just noted, these ligaments in fact never completely disappear.

\section{$5 \quad$ Perspectives}

Numerical solution of the integral equations (49) permits to determine, for given values of the geometrical parameters and the ratio $K_{I I I}^{(0)} / K_{I}^{(0)}$ of the initial SIF prior to propagation of the facets, the unknown tractions $p, q$, from which may be deduced: (i) via equations (47), the stresses $\Sigma_{Y Y}^{\mathrm{CZ}}, \Sigma_{Z Z}^{\mathrm{CZ}}, \Sigma_{Y Z}^{\mathrm{CZ}}$ on the $\mathrm{CZ}$ in the outer problem and therefore the remote stresses $\sigma_{11}^{\infty}=\Sigma_{Z Z}^{\mathrm{CZ}}, \sigma_{22}^{\infty}=\Sigma_{Y Y}^{\mathrm{CZ}}, \sigma_{12}^{\infty}=-\Sigma_{Y Z}^{\mathrm{CZ}}$ in the inner problem; (ii) via equations (51), the ratio $G / G^{(0)}$ of the global energy-release-rate to that in the absence of facets. This opens the way to various future applications of two distinct types briefly sketched here:

- In applications of local type, one may derive from the values of the remote stresses in the inner problem, combined with suitable criteria, the values of the geometrical parameters

$\overline{6}$ Note that the behaviors of the functions $f_{L}, g_{L}$ on the one hand, $f_{R}, g_{R}$ on the other hand, when their arguments $X / d,(a-X) / d$ go to zero, are different: $f_{L}, g_{L}$ diverge like the inverse square root of their argument (since $p, q$ diverge like $X^{-1 / 2}$ for small $X$ ) while $f_{R}, g_{R}$ go to finite limits (since $p, q$ take finite values at $X=a$ ). These different behaviors explain the different prefactors in the asymptotic expressions of these functions, $d^{-1 / 2}$ for $f_{L}, g_{L}, a^{-1 / 2}$ for $f_{R}, g_{R}$. 


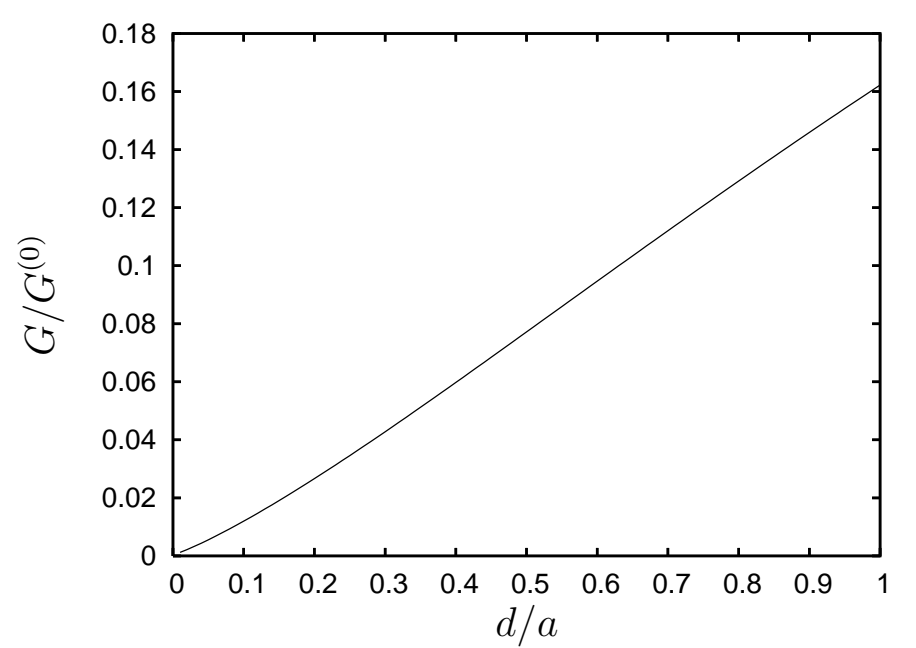

(a) The ratio $G / G^{(0)}$ as a function of $d / a$ for $c / d=0.6$

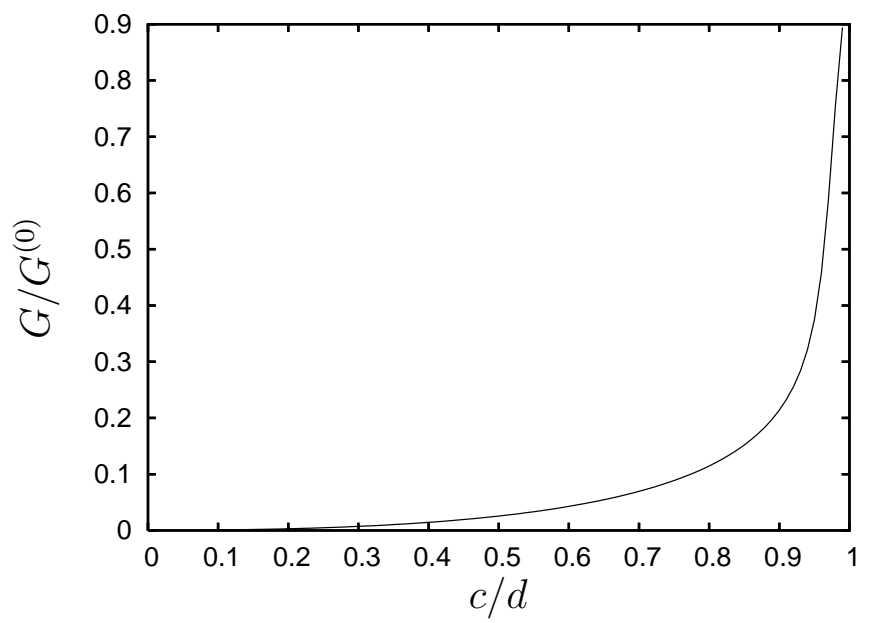

(b) The ratio $G / G^{(0)}$ as a function of $c / d$ for $d / a=0.3$

Fig. 8. The ratio $G / G^{(0)}$ for $\nu=0.3, K_{I I I}^{(0)} / K_{I}^{(0)}=0.15, \alpha=18.4^{\circ}$ and various values of $c / d$ and $d / a$

$\alpha$ and $c / d$ of the facets.

- For the prediction of the stationary tilt angle $\alpha$, one may request that the $2 \mathrm{D}$ SIF $k_{I I}$ at the tips of the $2 \mathrm{D}$ cracks in the inner problem, that is on the lateral sides of the facets, be zero. The underlying physical idea is that even though the facets essentially develop in the main direction of propagation, they must also extend laterally to some degree; and if the mode II SIF is nonzero on their lateral sides, kinking must inevitably occur, resulting in non-stationarity of the tilt angle.

- Another idea for the prediction of $\alpha$ would be to request that the total elastic energy be minimum with respect to this angle, the facet width being fixed. (The results of Section 2 permit to perform this minimization since they provide the variation of energy due to the cracks as a function of the various parameters). Such a procedure would be in line with Bourdin et al. (2000)'s variational theory of fracture, which has already been applied with some success to similar problems where the cracks may explore various positions and select the "optimal" one, see e.g. Maurini et al. (2013)'s study of drying of a colloidal suspension in a capillary tube.

- For the prediction of $c / d$, it would seem natural to assume that the facets extend 
laterally until the energy-release-rate on their lateral sides becomes less than the critical value. This idea can however work only if this energy-release-rate ultimately decreases when $c / d$ becomes close to unity, which is a somewhat debatable point since in this limit, in the expressions (6) of the $2 \mathrm{D}$ SIF $k_{I}, k_{I I}$ on the lateral sides of the facets, both the term $\sqrt{2 d \tan \left(\frac{\pi c}{2 d}\right)}$ and the functions $F_{i j}^{p}(c / d, \alpha)$ go to infinity, whereas the remote stresses go to zero due to the gradual opening of the CZ. Detailed inspection of the equations of the model, with the expressions of the functions $F_{i j}^{p}$ and the coefficients $\mathcal{A}_{\lambda \mu}$ provided in Appendices $\mathrm{A}$ and $\mathrm{B}$, reveals that it does predict that when $c / d$ becomes close to unity, the decrease of the remote stresses overcomes the increase of the term $\sqrt{2 d \tan \left(\frac{\pi c}{2 d}\right)}$ and the functions $F_{i j}^{p}$ so that $k_{I}$ and $k_{I I}$ ultimately decrease; but this happy conclusion cannot be regarded as final insofar as the formulae of Appendices A and B unfortunately become inaccurate precisely in the limit $c / d \rightarrow 1$ (for all nonzero values of $\alpha$ ).

- In applications of global type, one may calculate the critical intensity of the mode I+III loading ensuring propagation of the crack in the form of disconnected tilted facets, assuming the geometrical parameters of these facets to be known.

- One foreseeable conclusion will be that a larger mode I+III loading is required to propagate an array of tilted facets than a planar crack. This is because for a typically observed segmented crack front geometry, $c \sim d$ while $d / a<1$, thereby implying that $G / G^{(0)}<1$. In contrast, the ratio of the fractured area produced per unit length of facet advance to the one produced per unit length of planar crack advance is of order unity for typical values of the parameters $c / d$ and $\alpha$. Combination of these two elements implies a larger global fracture toughness for an array of daughter cracks than for a continuously connected parent crack.

- Another conclusion will pertain to the ineluctability of facet coarsening. Indeed in the absence of coarsening, equations (49) apply with a continuously decreasing ratio $d / a$ as propagation proceeds, since the half-spacing $d$ of the facets remains constant while their length $a$ increases. The results of Subsection 4.5 have shown that $G$ decreases to zero then, implying that propagation in the form of disconnected tilted facets must, under constant loading, stop. In contrast, with facet coarsening, the relevant equations are (52). Even without solving these equations numerically, one can easily see that if $d$ increases linearly with $X$, they can be put in non-dimensional form in such a way as to become independent of $a$; then $G$ no longer depends on the distance of propagation, thereby allowing for the possibility of sustained propagation in the form of disconnected tilted facets under constant loading. ${ }^{7}$

\section{Conclusion}

The aim of this paper was to develop a macroscopic model of crack propagation in mode I+III in the form of disconnected, tilted facets by schematizing the region of these facets

\footnotetext{
$\overline{7}$ The linear dependence of $d$ upon $X$ is in fact the only one warranting such a condition; it may indeed be checked that if $d$ increases, more generally, like $X^{\gamma}$ where $\gamma$ is some positive exponent, $G$ is independent of $a$ if and only if $\gamma$ is unity.
} 
as a CZ. The development was based on the fundamental assumption of smallness of the facet spacing as compared to the facet length. Three major steps were necessary:

- A preliminary one consisted of complementing the solution for a periodic array of inclined cracks by calculating the variations of remote displacements due to presence of the cracks, needed to define the constitutive law of the CZ. Leblond and Frelat (2014)'s approximate solution provided only the SIF but the required variations were deduced from there by using the classical compliance formula.

- In a second step, the constitutive law of the $\mathrm{CZ}$ was derived from a matched asymptotic expansion procedure, using the ratio of the facet spacing to the facet length as a small parameter. The major conclusion of this procedure was that the relations connecting the displacement discontinuities on the $\mathrm{CZ}$ to the local stresses, in the outer problem, are identical to those connecting the variations of far displacements due to presence of the cracks to the far stresses, in the inner one, and hence may be deduced from the results of the first step.

- In a third step, 1D integral equations on the unknown tractions acting on the CZ, defining the model looked for, were derived by eliminating the displacement discontinuities between the constitutive law of the $\mathrm{CZ}$ and their integral expressions in terms of these tractions. A strategy for numerical solution of these equations was proposed, and numerical examples were provided.

Future applications of the model to prediction of both local aspects of crack propagation in the form of disconnected, tilted facets (tilt angle of the facets, ratio of their width to their spacing) and global ones (critical intensity of the loading promoting propagation, necessity of facet coarsening) were briefly discussed in conclusion.

\section{Acknowledgements}

The authors wish to thank Vincent Hakim, of Ecole Normale Superieure, for illuminating discussions. The financial support of Institut Universitaire de France (JBL and VL), ANR GeoSMEC, contract 2012-BS06-0016-03 (VL) and US DOE grant DEFG02-07ER46400 $(\mathrm{AK})$ is also gratefully acknowledged.

\section{References}

Bourdin B., Francfort G.A. and Marigo J.J. (2000). Numerical experiments in revisited brittle fracture. J. Mech. Phys. Solids, 48, 797-826.

Cooke M.L. and Pollard D.D. (1996). Fracture propagation paths under mixed-mode loading within rectangular blocks of polymethyl methacrylate. J. Geophys. Res., 101, 3387-3400.

Cotterell B. and Rice J.R. (1980). Slightly curved or kinked cracks. Int. J. Fracture, 16, 155-169.

Fleck N. (1991). Brittle fracture due to an array of microcracks. Proc. Roy. Soc. London A, 432, 55-76. 
Gao H. and Rice J.R. (1986). Shear stress intensity factors for planar crack with slightly curved front. ASME J. Appl. Mech., 53, 774-778.

Goldstein R.V. and Osipenko N.M. (2012). Successive development of the structure of a fracture near the front of a longitudinal shear crack. Doklady Physics, 57, 281-284.

Goldstein R.V. and Salganik R.L. (1974). Brittle fracture of solids with arbitrary cracks. Int. J. Fracture, 10, 507-523.

Griffith A.A. (1921). The phenomena of rupture and flow in solids. Phil. Trans. Roy. Soc. London, Series A, 221, 163-198.

Gradshteyn I.S. and Ryzhik I.M. (1980). Table of Integrals, Series, and Products, Academic Press, New York.

Hakim V. and Karma A. (2009). Laws of crack motion and phase-field models of fracture. J. Mech. Phys. Solids, 57, 342-368.

Hourlier F. and Pineau A. (1979). Fissuration par fatigue sous sollicitations polymodales (mode I ondulé + mode III permanent) d'un acier pour rotors 26NCDV14. Mémoires Scientifiques de la Revue de Métallurgie, 76, 175-185 (in French).

Karma A., Kessler D.A. and Levine H. (2001). Phase-field model of mode III dynamic fracture. Phys. Rev. Lett., 87, 045501 [4 pages].

Knauss W.G. (1970). An observation of crack propagation in antiplane shear. Int. J. Fracture, 6, 183-187.

Koiter W.T. (1959). An infinite row of parallel cracks in an infinite elastic sheet. IngenieurArchiv, 28, 168-172.

Lazarus V. (1997). Quelques problèmes tridimensionnels de mécanique de la rupture fragile. Ph.D. Thesis, Université Pierre et Marie Curie (Paris VI), France (in French).

Lazarus V., Buchholz F.G., Fulland M. and Wiebesiek J. (2008). Comparison of predictions by mode II or mode III criteria on crack front twisting in three or four point bending experiments. Int. J. Fracture, 153, 141-151.

Lazarus V., Leblond J.B. and Mouchrif S.E. (2001b). Crack front rotation and segmentation in mixed-mode I+III or I+II+III - Part II: Comparison with experiments, $J$. Mech. Phys. Solids, 49, 1421-1443.

Leblond J.B., Frelat J. (2014). Development of fracture facets from a crack loaded in mode I+III: solution and application of a model 2D problem, J. Mech. Phys. Solids, 64, 133-153.

Leblond J.B., Karma A. and Lazarus V. (2011). Theoretical analysis of crack front instability in mode I+III. J. Mech. Phys. Solids, 59, 1872-1887.

Leblond J.B. and Lazarus V. (2015). On the strong influence of imperfections upon the quick deviation of a mode I+III crack from coplanarity. To appear in J. Mech. Materials Structures.

Leguillon D. (1993). Asymptotic and numerical analysis of crack branching in nonisotropic materials. Eur. J. Mech. A/Solids, 12, 3351.

Lin B., Mear M.E. and Ravi-Chandar K. (2010). Criterion for initiation of cracks under mixed-mode I+III loading. Int. J. Fracture, 165, 175-188.

Maurini C., Bourdin B., Gauthier G. and Lazarus, V. (2013). Crack patterns obtained by unidirectional drying of a colloidal suspension in a capillary tube: experiments and numerical simulations using a two-dimensional variational approach. Int. J. Fracture, 184, 75-91.

Melin S. (1983). Why do cracks avoid each other? Int. J. Fracture, 23, 37-45.

Movchan A.B., Gao H. and Willis J.R. (1998). On perturbations of plane cracks. Int. J. 
Solids Structures, 35, 3419-3453.

Muskhelishvili N.I. (1953). Some Basic Problems of the Mathematical Theory of Elasticity, Noordhoff, Groningen.

Palaniswamy K. and Knauss W.G. (1975). Crack extension in brittle solids. In: Mechanics Today, Vol. 4, Nemat-Nasser, Ed., Pergamon Press, pp. 87-148.

Pham K.H. and Ravi-Chandar K. (2014). Further examination of the criterion for crack initiation under mixed-mode I+III loading. Int. J. Fract., 189, 121-138.

Pollard D.D. and Aydin A. (1988). Progress in understanding jointing over the past century. Geol. Soc. Amer. Bull., 100, 1181-1204.

Pollard D.D., Segall P. and Delaney P.T. (1982). Formation and interpretation of dilatant echelon cracks. Geol. Soc. Amer. Bull., 93, 1291-1303.

Pons A.J. and Karma A. (2010). Helical crack-front instability in mixed-mode fracture. Nature, 464, 85-89.

Ronsin O., Caroli C. and Baumberger T. (2014). Crack front echelon instability in mixedmode fracture of a strongly nonlinear elastic solid. Europhys. Lett., 105, 34001 (6 pages).

Sanchez-Hubert J. and Sanchez-Palencia E. (1992). Introduction aux méthodes asymptotiques et à l'homogénéisation, Masson, Paris.

Sommer E. (1969). Formation of fracture "lances" in glass. Engng. Fracture Mech., 1, 539-546.

Suresh S. and Tschegg E.K. (1987). Combined mode I - mode III fracture of fatigueprecracked alumina. J. Amer. Ceramic Soc., 70, 726-733.

Yates J.R. and Miller K.J. (1989). Mixed-mode (I+III) fatigue thresholds in a forging steel. Fatigue Fracture Engng. Materials Structures, 12, 259-270. 


\section{A Appendix: approximate expressions of the functions $F_{i j}^{p}$}

The approximate expressions of the functions $F_{i j}^{p}(c / d, \alpha)$ proposed by Leblond and Frelat (2014) (in the sole case $c<d$ ) are as follows:

$$
\left\{\begin{array}{l}
F_{11}^{I}\left(\frac{c}{d}, \alpha\right) \simeq \frac{1-\cos (2 \alpha)}{2 \sqrt{\cos \alpha}} ; \quad F_{22}^{I}\left(\frac{c}{d}, \alpha\right) \simeq \frac{1+\cos (2 \alpha)}{2 \sqrt{\cos \alpha}} ; \\
F_{12}^{I}\left(\frac{c}{d}, \alpha\right) \simeq-\left[3+\frac{\pi c / d}{\sin (\pi c / d)}\right] \frac{\sin (2 \alpha)}{4 \sqrt{\cos \alpha}} ; \\
F_{11}^{I I}\left(\frac{c}{d}, \alpha\right) \simeq-\frac{\sin (2 \alpha)}{2 \sqrt{\cos \alpha}} ; \quad F_{22}^{I I}\left(\frac{c}{d}, \alpha\right) \simeq\left[3-\frac{\pi c / d}{\sin (\pi c / d)}\right] \frac{\sin (2 \alpha)}{4 \sqrt{\cos \alpha}} \\
F_{12}^{I I}\left(\frac{c}{d}, \alpha\right) \simeq \frac{\cos (2 \alpha)}{\sqrt{\cos \alpha}}
\end{array}\right.
$$

These formulae present the following nice features:

- for small values of $\alpha$ but arbitrary values of $c / d(<1)$, they match the exact first-order solution in $\alpha$ (Melin, 1983; Leblond and Frelat, 2014);

- for infinitesimal values of $c / d$ but arbitrary values of $\alpha$, they again match the exact, trivial solution corresponding to isolated cracks;

- they yield acceptable results in all cases except when $\alpha$ and $c / d$ are simultaneously large (see Leblond and Frelat (2014)'s comparisons with the results of some finite element calculations).

\section{B Appendix: approximate expressions of the coefficients $\mathcal{A}_{\lambda \mu}$}

Approximate expressions of the coefficients $\mathcal{A}_{\lambda \mu}$ may be derived using their definition (18) and the approximate expressions (A.1) of the functions $F_{i j}^{p}$. All integrals involved reduce to elementary integrals plus a single, non-elementary one defined by

$$
J(u) \equiv \int_{0}^{u} \frac{v^{2}}{\sin v} \mathrm{~d} v
$$

but practical calculation of this integral does not raise any problem since it is given by the following very quickly converging series (Gradshteyn and Ryzhik (1980), formula 2.643.3):

$$
J(u)=\frac{u^{2}}{2}+\sum_{k=1}^{+\infty}(-1)^{k+1} \frac{2^{2 k-1}-1}{(k+1)(2 k) !} B_{2 k} u^{2(k+1)}
$$

where $B_{i}$ is the $i$-th Bernoulli number.

Defining

$$
\bar{x} \equiv \frac{\pi x}{2},
$$


the expressions found are as follows:

$$
\begin{aligned}
& \mathcal{A}_{11}(x, \alpha) \simeq-\frac{2}{\pi} \tan ^{2} \alpha \ln (\cos \bar{x}) \\
& \mathcal{A}_{22}(x, \alpha) \simeq \frac{1}{2 \pi \cos ^{2} \alpha}\left\{-\ln (\cos \bar{x})\left[-3 \cos ^{2}(2 \alpha)+2 \cos (2 \alpha)+5\right]\right. \\
& \left.+\left[-\frac{7}{4} \bar{x} \tan \bar{x}+\frac{\bar{x}^{2}}{8 \cos ^{2} \bar{x}}+\frac{J(2 \bar{x})}{16}\right] \sin ^{2}(2 \alpha)\right\} \\
& \mathcal{A}_{33}(x, \alpha) \simeq \frac{1}{2 \pi \cos ^{2} \alpha}\left\{-\ln (\cos \bar{x})\left[3 \cos ^{2}(2 \alpha)+1\right]\right. \\
& \left.+\left[\frac{5}{4} \bar{x} \tan \bar{x}+\frac{\bar{x}^{2}}{8 \cos ^{2} \bar{x}}+\frac{J(2 \bar{x})}{16}\right] \sin ^{2}(2 \alpha)\right\} \\
& \mathcal{A}_{12}(x, \alpha)=\mathcal{A}_{21}(x, \alpha) \simeq \frac{2}{\pi} \sin ^{2} \alpha\left[\ln (\cos \bar{x})+\frac{\bar{x}}{2} \tan \bar{x}\right] ; \\
& \mathcal{A}_{13}(x, \alpha)=\mathcal{A}_{31}(x, \alpha) \simeq \frac{\tan \alpha}{\pi}\left\{\ln (\cos \bar{x})[\cos (2 \alpha)+1]+\frac{\bar{x}}{2} \tan \bar{x}[\cos (2 \alpha)-1]\right\} ; \\
& \mathcal{A}_{23}(x, \alpha)=\mathcal{A}_{32}(x, \alpha) \simeq \frac{\tan \alpha}{\pi}\left\{\ln (\cos \bar{x})[-3 \cos (2 \alpha)+1]-\frac{\bar{x}}{2} \tan \bar{x}[3 \cos (2 \alpha)+1]\right\} \text {. }
\end{aligned}
$$

\section{Appendix: expressions of the coefficients $M_{i j}$ and $N_{i j}$}

The definitions (59) of the coefficients $M_{i j}$ and $N_{i j}$ may be rewritten in the form

$$
\left\{\begin{array}{lll}
M_{i j} \equiv \phi\left(w_{j-1}^{\prime}, w_{j}^{\prime} ; w_{i}\right) & , \quad \phi(u, v ; w) \equiv P V \int_{u}^{v} \sqrt{\frac{1-w^{\prime}}{w^{\prime}}} \frac{\mathrm{d} w^{\prime}}{w^{\prime}-w} \\
N_{i j} \equiv \psi\left(w_{j-1}^{\prime}, w_{j}^{\prime} ; w_{i}\right) & , \quad \psi(u, v ; w) \equiv P V \int_{u}^{v} \sqrt{w^{\prime}\left(1-w^{\prime}\right)} \frac{\mathrm{d} w^{\prime}}{w^{\prime}-w}
\end{array}\right.
$$

These integrals may be reduced to ordinary, easily calculable integrals plus a single one in principal value, by using the change of variable $w^{\prime}=\sin ^{2} \theta$ and then expanding the numerator in powers of $\sin ^{2} \theta-w$; one thus gets

$$
\left\{\begin{aligned}
\phi(u, v ; w)= & 2[\arcsin (\sqrt{u})-\arcsin (\sqrt{v})]+2(1-w) \chi(u, v ; w) \\
\psi(u, v ; w)= & (1-2 w)[\arcsin (\sqrt{v})-\arcsin (\sqrt{u})]+\sqrt{v(1-v)}-\sqrt{u(1-u)} \\
& +2 w(1-w) \chi(u, v ; w)
\end{aligned}\right.
$$

where

$$
\chi(u, v ; w) \equiv P V \int_{\arcsin (\sqrt{u})}^{\arcsin (\sqrt{v})} \frac{\mathrm{d} \theta}{\sin ^{2} \theta-w}
$$


The problem is thus reduced to calculating the single integral in principal value $\chi(u, v ; w)$, which is easily done by using Gradshteyn and Ryzhik (1980)'s formula (2.562.1); the three cases $w<\arcsin (\sqrt{u}), \arcsin (\sqrt{u})<w<\arcsin (\sqrt{v}), \arcsin (\sqrt{v})<w$ must be distinguished in the calculation but the results may be expressed in a single formula:

$$
\chi(u, v ; w)=\frac{\Xi(u ; w)-\Xi(v ; w)}{\sqrt{w(1-w)}} \quad, \quad \Xi(t ; w) \equiv \begin{cases}\operatorname{argtanh}\left(\sqrt{\frac{t(1-w)}{w(1-t)}}\right) & \text { if } t<w \\ \operatorname{argcoth}\left(\sqrt{\frac{t(1-w)}{w(1-t)}}\right) & \text { if } t>w .\end{cases}
$$

Formulae (C.1), (C.2) and (C.4) provide the desired expressions of the coefficients $M_{i j}$ and $N_{i j}$. 